\title{
A seven-year surveillance of epidemiology of malaria reveals travel and gender are the key drivers of dispersion of drug resistant genotypes in Kenya
}

\author{
Moureen N. Maraka ${ }^{\text {Corresp., } 1,2}{ }^{2}$, Hoseah Akala ${ }^{2}$, Asito Amolo ${ }^{1}$, Dennis Juma ${ }^{2}$, Duke Omariba ${ }^{2}$, Agnes Cheruiyot ${ }^{2}$, \\ Benjamin Opot ${ }^{2}$, Charles Okudo ${ }^{2}$, Edwin Mwakio ${ }^{2}$, Gladys Chemwor ${ }^{2}$, Jackline Juma ${ }^{2}$, Raphael Okoth ${ }^{2}$, \\ Redemptah Yeda ${ }^{2}$, Ben Andagalu ${ }^{\text {Corresp. } 2}$ \\ ${ }^{1}$ School of Health Sciences, Jaramogi Oginga Odinga University of Science and Technology., Bondo, Siaya, Kenya \\ 2 Department of Emerging Infectious Diseases (DEID), United States Army Medical Research Directorate-Africa Kenya (USAMRD-A Kenya)/Kenya Medical \\ Research Institute (KEMRI), Kisumu, Kisumu, Kenya \\ Corresponding Authors: Moureen N. Maraka, Ben Andagalu \\ Email address: maraka204@gmail.com, Ben.Andagalu@usamru-k.org
}

Malaria drug resistance is a global public health concern. Though parasite mutations have been associated with resistance, other factors could influence the resistance. A robust surveillance system is required to monitor and help contain the resistance. This study established the role of travel and gender in dispersion of chloroquine resistant genotypes in malaria epidemic zones in Kenya. 1,776 individuals presenting with uncomplicated malaria at hospitals selected from four malaria transmission zones in Kenya between 2008 and 2014 were enrolled in a prospective surveillance study assessing the epidemiology of malaria drug resistance patterns. Demographic and clinical information per individual was obtained using a structured questionnaire. Further, $2 \mathrm{~mL}$ of blood was collected for malaria diagnosis, parasitemia quantification and molecular analysis. DNA extracted from dried blood spots collected from each of the individuals was genotyped for polymorphisms in Plasmodium falciparum chloroquine transporter gene (Pfcrt 76), Plasmodium falciparum multidrug resistant gene 1 (Pfmdr1 86 and Pfmdr1 184) regions that are putative drug resistance genes using both conventional polymerase chain reaction (PCR) and real-time PCR. The molecular and demographic data was analyzed using Stata version 13 (College Station, TX: StataCorp LP) while mapping of cases at the selected geographic zones was done in ArcGIS version 10. Chloroquine resistant (CQR) genotypes across gender revealed an association with chloroquine resistance by both univariate model $(p=0.027)$ and by multivariate model $(p=0.025)$, female as reference group in both models. Prior treatment with antimalarial drugs within the last 6 weeks before enrollment was associated with carriage of CQR genotype by multivariate model $(p=0.034)$. Further, a significant relationship was observed between travel and CQR carriage both by univariate model ( $p=$ $0.001)$ and multivariate model $(p=0.002)$. These findings suggest that gender and travel 
are significantly associated with chloroquine resistance. From a gender perspective, males are more likely to harbor resistant strains than females hence involved in strain dispersion. On the other hand, travel underscores the role of transport network in introducing spread of resistant genotypes, bringing in to focus the need to monitor gene flow and establish strategies to minimize the introduction of resistance strains by controlling malaria among frequent transporters. 


\section{A seven-year surveillance of epidemiology of malaria}

2 reveals travel and gender are the key drivers of dispersion

3 of drug resistant genotypes in Kenya.

4

5 Moureen N. Maraka ${ }^{12}$, Hoseah M. Akala ${ }^{2}$, Asito Amolo ${ }^{1}$, Dennis Juma ${ }^{2}$, Duke Omariba ${ }^{2}$, Agnes

6 Cheruiyot $^{2}$, Benjamin Opot ${ }^{2}$, Charles Okudo ${ }^{2}$, Edwin Mwakio $^{2}$, Gladys Chemwor ${ }^{2}$, Jackline

7 Juma $^{2}$, Raphael Okoth ${ }^{2}$, Redemptah Yeda and Ben Andagalu ${ }^{2}$

8

$9{ }^{1}$ School of Health Sciences, Jaramogi Oginga Odinga University of Science and Technology,

10 Bondo, Siaya County, Kenya.

$11{ }^{2}$ Department of Emerging Infectious Diseases (DEID), United States Army Medical Research

12 Directorate-Africa Kenya (USAMRD-A Kenya)/Kenya Medical Research Institute (KEMRI),

13 Kisumu, Kisumu County Kenya.

Corresponding Authors:

16 Moureen N. Maraka ${ }^{12}$,

$17{ }^{1}$ P.O. Box 210 - 40601 Bondo - Kenya.

18254 Off Kisumu-Busia Road, Kisumu, Kisumu County, 40100, Kenya.

19 Email address: maraka204@gmail.com

20

21

Ben Andagalu ${ }^{2}$

22

54 Off Kisumu-Busia Road, Kisumu, Kisumu County, 40100, Kenya.

Email address: Ben.Andagalu@usamru-k.org

24

25

26

27

28

29

30

31

32

33 


\section{Abstract}

40 Malaria drug resistance is a global public health concern. Though parasite mutations have been associated with resistance, other factors could influence the resistance. A robust surveillance system is required to monitor and help contain the resistance. This study established the role of travel and gender in dispersion of chloroquine resistant genotypes in malaria epidemic zones in

44 45

46 Kenya. 1,776 individuals presenting with uncomplicated malaria at hospitals selected from four malaria transmission zones in Kenya between 2008 and 2014 were enrolled in a prospective surveillance study assessing the epidemiology of malaria drug resistance patterns. Demographic and clinical information per individual was obtained using a structured questionnaire. Further, $2 \mathrm{~mL}$ of blood was collected for malaria diagnosis, parasitemia quantification and molecular analysis. DNA extracted from dried blood spots collected from each of the individuals was genotyped for polymorphisms in Plasmodium falciparum chloroquine transporter gene (Pfcrt 76), Plasmodium falciparum multidrug resistant gene 1 (Pfmdr1 86 and Pfmdr1 184) regions that are putative drug resistance genes using both conventional polymerase chain reaction (PCR) and real-time PCR. The molecular and demographic data was analyzed using Stata version 13 (College Station, TX: StataCorp LP) while mapping of cases at the selected geographic zones was done in ArcGIS version 10. Chloroquine resistant (CQR) genotypes across gender revealed an association with chloroquine resistance by both univariate model $(p=0.027)$ and by multivariate model $(p=0.025)$, female as reference group in both models. Prior treatment with antimalarial drugs within the last 6 weeks before enrollment was associated with carriage of CQR genotype by multivariate model $(p=0.034)$. Further, a significant relationship was observed between travel and CQR carriage both by univariate model $(p=0.001)$ and multivariate model $(p=$ 0.002). These findings suggest that gender and travel are significantly associated with chloroquine resistance. From a gender perspective, males are more likely to harbor resistant strains than females hence involved in strain dispersion. On the other hand, travel underscores the role of transport network in introducing spread of resistant genotypes, bringing in to focus the 
65

\section{Introduction}

68 In 2006, the World Health organization (WHO) recommended artemisinin-based combination 69

need to monitor gene flow and establish strategies to minimize the introduction of resistance strains by controlling malaria among frequent transporters. therapy (ACTs) use as first-line drug for treatment of uncomplicated malaria following widespread failure of sulfadoxine-pyrimethamine combination treatment. The strategy relied on the fast acting artemisinin derivatives rapidly to bring down the parasite biomass, relieves symptoms while the partner drug with longer half-life clears the residual parasites therefore cushioning the artemisinin derivative from emerging resistance (Nosten \& White, 2007). The most widely used combinations globally include artemether-lumefantrine (AL, Coartem), dihydroartemisinin-piperaquine (DHAPPQ), artesunate-amodiaquine (ASAQ) and artesunatemefloquine. These treatments alongside other intervention have contributed to about $37 \%$ decline in malaria cases and 60\% reduction in mortality during the last fifteen years (Dhiman, 2019). However, the recent reports of resistance to artemisinin derivative of the ACTs in Southeast Asia (Woodrow \& White, 2017) and its pattern of migration (Shetty et al., 2019) threaten this trajectory due to fears that the global dissemination of these strains would follow historical pattern of drug resistant strain dispersal. Africa parasite diversity studies show that these strains have not yet been detected in Africa (Kamau et al., 2015), but shows extant subpopulations of P. falciparum in sub-Saharan Africa (Amambua-Ngwa et al., 2019) suggestive of parallel emergence of ACT resistance in the region given the varying first-line drugs in across Africa. Notwithstanding the origin of the initial resistance, it is essential to understand patterns of the dispersal present-day characterized strains in natural infections at local level as a stop-gap for containment should ACT resistant strains emerge.

Non-synonymous mutations in the kelch propeller domain (K13-propeller) in Plasmodium falciparum have been associated with artemisinin resistance in samples from Southeast Asia (Witkowski et al., 2013). Further, the waning efficacy of ACTs has been partly associated with failure of the quinoline partners in the combination (Borrmann, Matsiegui, Missinou, \& Kremsner, 2008). Though most of these drugs have not been policy recommended, crossresistance has been shown to occur owing to similarity in mechanisms of actions (Veiga et al., 2016). A case in point is the diminished mefloquine sensitivity in Kenya (Eyase et al., 2013) yet 
95 mefloquine had neither been first-line nor second-line treatment in the country. Single

96 Nucleotide Polymorphisms (SNPs) in the P. falciparum multidrug resistance protein (Pfmdrl)

97 and $P$. falciparum chloroquine resistance transporter $(P f c r t)$ genes confer resistance to a number

98 of anti-malaria drugs. Chloroquine resistance has been associated to Pfcrt K76T SNP

99 (Mohammed et al., 2013; Zhao et al., 2019). Pfmdr1 86Y and Y184 haplotypes have been shown 100 to modulate chloroquine (CQ) resistance in the presence of Pfcrt 76T mutation (Venkatesan et

101 al., 2014). Mefloquine (MQ) and lumefantrine (LU) sensitivities are linked to Pfmdr1 86Y point 102 mutation. Additionally, emerging Pfcrt K76 allele carrying parasites carrying Pfmdr1 N86 and

$103184 \mathrm{~F}$ in the last decade have been linked to declining susceptibility to LU, part of the AL

104 treatment that is recommended in Kenya (Achieng et al., 2015; Eyase et al., 2013; Venkatesan et

$105 a l ., 2014)$, and a reciprocal rise in parasite susceptibility to CQ and amodiaquine (ADQ)

106 (Achieng et al., 2015; Veiga et al., 2016). Conversely, our previous studies showed that the

107 prevalence of Pfcrt K76 and N86 increased from 6.4\% in 1995-1996 to $93.2 \%$ in 2014 and $0.0 \%$

108 in 2002-2003 to 92.4\% in 2014 respectively (Achieng et al., 2015), a period when the first and

109 second-line malaria drugs for Kenya are AL and DHAPPQ respectively. A study done in

110 Zanzibar reported that treatment with AL selects for chloroquine-susceptible Pfcrt K76 allele

111 while re-infecting parasites harbor Pfmdr1 N86 and 184F alleles (Sisowath et al., 2009).

112 Development of resistance is often attributed to acquisition of survival advantage of parasites

113 against drugs (Hastings \& Donnelly, 2005). The rate and pattern of spread of initial resistant

114 strain is often dependent on multiple confounding factors that should be monitored alongside

115 drug resistant surveillance programs concurrently (Mbugi et al., 2006). The confounding factors

116 includes initial prevalence of mutations, population movement between transmission regions among

117 others (Bloland, 2001). Distribution, control and prevention of malaria has been reported to be

118 influenced by human population movement (HPM) from areas with high transmission rates to

119 malaria free areas (Cohen et al., 2012). Similarly, HPM has been shown to contribute to spread

120 of drug resistant parasite strains elsewhere (Lynch \& Roper, 2011). Other factors like age,

121 gender, location, history of past malaria infection and previous malaria treatment have been

122 shown to affect malaria prevention and control. These factors could consequently be associated

123 with the dispersal and distribution of chloroquine resistance.

124 To combat drug resistance, the WHO recommends a robust surveillance system to monitor and 125 contain the drug resistance (WHO, 2009). In areas with documented success and declining 
126 disease burden, there are calls for initiation of pre-elimination/elimination phase in malaria 127 control and management (Whittaker, Dean, \& Chancellor, 2014; WHO, 2015). However, 128 elimination process is facing numerous challenges. A study done in Nigeria reported research, 129 political will/funding, attitude/behavior change, conflicts, terrorism/migration, climate change, 130 insecticide resistance, drug resistance and treatment as challenges facing malaria elimination 131 (Aribodor D.N, Ugwuanyi I.K., \& Aribodor O.B, 2016), highlighting the importance of 132 understanding the malaria drug resistance patterns alongside demographics/clinical factors and 133 parasite characteristics among symptomatic malaria cases as a prerequisite for entry in pre134 elimination phase.

135 As countries move towards the elimination phase, enhanced surveillance systems are required to ensure that every infection is detected, treated and reported (WHO, 2016a). However, there is lack of sufficient information to feed such a trend of reduced malaria burden in Kenya. Most studies done in Kenya often dwell on one site mostly endemic regions, with a smaller sample size and no detailed information on demographics. Therefore, this study aimed at determining the role of gender and travel in dispersion of chloroquine resistant genotypes among individuals with symptomatic malaria in epidemic zones, Kenya.

\section{Materials \& Methods}

143 Ethics Statement, Study Protocol, Sites and Subjects: This study was approved by the Kenya 144 Medical Research Institute (KEMRI), Walter Reed Army Institute of Research (WRAIR) and

145 Human Research Protection office institutional review boards (protocol numbers: KEMRI 146 \#1330, WRAIR \#1384 and HRPO Log \#A-19306.3) respectively. Seven participating clinical 147 centers were all Ministry of Health facilities located in various malaria epidemic regions in

148 Kenya (Fig. 1); Lake endemic region (Kisumu East and Kisumu West/Kombewa District 149 Hospitals) both situated in Kisumu County (0¹4'60.00" N 3454'59.99" E), Highland epidemic region had (Kericho District Hospitals and Kisii teaching/referral hospital) situated in Kericho

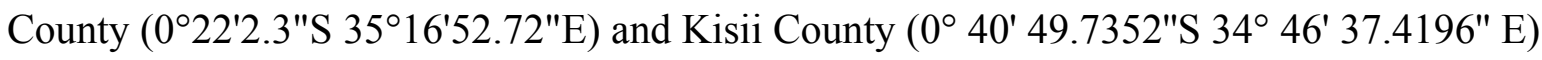
respectively, Coast endemic region (Malindi District Hospital) situated in Kilifi County ( $\left.3^{\circ} 13^{\prime} 25^{\prime \prime} \mathrm{S} 40^{\circ} 7^{\prime} 48^{\prime \prime} \mathrm{E}\right)$ and Semiarid seasonal region (Isiolo District, Isiolo county $\left(0^{\circ} 52^{\prime} 60.00^{\prime \prime} \mathrm{N}\right.$ $\left.38^{\circ} 40^{\prime} 0.12^{\prime \prime} \mathrm{E}\right)$ and Marigat Sub district Hospital, Baringo County ( $\left.0^{\circ} 28^{\prime} \mathrm{N} 35^{\circ} 59^{\prime} \mathrm{E}\right)$. At each participating facility, training of medical staff, capacity building, and facility upgrades were 
156 provided by the Global Emerging Infections Surveillance (GEIS) Program, U.S. Department of 157 Defense. Subjects attending outpatient clinics from 2008 to 2014, at least 6 months old and 158 suspected of having non-complicated P. falciparum malaria were invited to participate. Written informed consent was obtained from adult subjects ( $\geq 18$ years of age) or legal guardians for subjects $<18$ years of age. Infants weighing less than 5 kilograms were excluded. Sample Collection and Preparation: $2 \mathrm{ml}$ of blood was collected from eligible subjects who had tested positive by rapid diagnostic test (RDT; Parascreen H (Pan/Pf), Zephyr Biomedicals, Verna Goa, India) for P. falciparum malaria. Additionally, FTA filter paper (Whatman Inc., Bound Brook, New Jersey, USA) was used to collect three blood spots of about $100 \mu 1$ each for P. falciparum DNA extraction and molecular analysis. Two blood films on glass slides were made for Giemsa staining at the laboratory for microscopic examination, to confirm RDT results and determine parasitemia. For discrepancies between RDT and microscopy, microscopy determined the final result. Collected specimens for molecular analysis were stored in $-20{ }^{\circ} \mathrm{C}$ freezers at the respective hospitals temporarily, before being transported to the KEMRI/USAMRD-A Kenya research laboratory in Kisumu for long-term storage at $-80^{\circ} \mathrm{C}$. The remainder $\sim 1.5 \mathrm{~mL}$ of blood was cryopreserved in liquid nitrogen for future culture and in vitro 172 susceptibility testing.

Subjects' demographic information, malaria history, symptoms for current infections and physical examination findings were recorded in case report forms (CRFs). Subjects were treated with oral artemether-lumefantrine (AL; Coartem) administered over three consecutive days, a standard of care for $P$. falciparum malaria in Kenya. The first dose was observed by the study team and remaining doses were self-administered at home.

Microscopy: Thick and thin smears were prepared for low and high parasitemia respectively. The smears were stained in fresh working Giemsa stain (diluted 1:10 in buffered water from stock) for 15-20 minutes and air dried. The parasite count was recorded as parasites per 200 white blood cells (WBCs) for thick smear and per 2000 red blood cells for the thin smear. The two were standardized to parasites per microliter. An assumed WBCs count of $8000 / \mu 1$ that has been accepted as reasonably accurate in estimating malaria parasite densities was used (Afrane, Zhou, Githeko, \& Yan, 2014; Gyasi et al., 2015). To convert parasite count per 2000 RBCs to parasites $/ \mu 1$ an assumed $\mathrm{RBCs}$ count of $4.5 \mathrm{X} 10^{\wedge} 6 / \mu \mathrm{L}$ was used (Desai et al., 2015). 
186 DNA extraction: DNA was extracted from blood spotted onto FTA filter papers using the 187 QIAamp DNA Mini Kit (QIAGEN sciences, Maryland, USA) in accordance with the 188 manufacturer's protocol for dried blood spots. The purified DNA was stored at $-20{ }^{\circ} \mathrm{C}$ until 189 required.

190 Pfcrt K76T SNP analysis: A fragment of Pfcrt gene spanning K76T SNP was amplified by 191 conventional polymerase chain reaction (PCR) on an Applied Biosystems' GenAmp PCR system

192 193 194

195

196

197

198

199

200

201

202

203

204

205

206

207

208

209

210

211

212

213

214

215

216 9700 (Foster City, CA, USA). The primer sequences used for the analysis are as described in (Wang et al., 2005). The primers were purchased from Applied Biosystems (Foster City, CA, USA). The primary reaction was performed in a $15 \mu \mathrm{L}$ reaction mixture which consisted of 0.5 $\mu \mathrm{L}$ AmpliTaq Gold (Applied Biosystems, Foster City, CA, USA), $2.5 \mu \mathrm{L}$ of $10 \mu \mathrm{M}$ forward primer and reverse primers, $2.5 \mu \mathrm{L}$ of $10 \mathrm{X}$ PCR Buffer, $2.5 \mu \mathrm{L}$ of $10 \mathrm{mM}$ dNTPs, $2.0 \mu \mathrm{L}$ of $25 \mathrm{Mm} \mathrm{MgCl}_{2}, 1 \mu \mathrm{L}$ of DNA sample, and $4 \mu \mathrm{L}$ water. The primary amplification was performed at the following conditions: $94{ }^{\circ} \mathrm{C}, 3$ minutes for initial denaturation, $94{ }^{\circ} \mathrm{C}, 30$ seconds for secondary denaturation and 45 cycles consisting of $56^{\circ} \mathrm{C}, 30$ seconds annealing, $60{ }^{\circ} \mathrm{C}$ for 30 seconds elongation followed by $64{ }^{\circ} \mathrm{C}$ for 3 minutes of final elongation and held at $4{ }^{\circ} \mathrm{C}$. The primary PCR amplicon was then used as a template in the secondary PCR. The Secondary reaction was performed in a $25 \mu \mathrm{L}$ reaction mixture which consisted of $0.5 \mu \mathrm{L}$ AmpliTaq Gold (Applied Biosystems, Foster City, CA, USA), $2.5 \mu \mathrm{L}$ of $1 \mu \mathrm{M}$ forward primer and reverse primers, $2.5 \mu \mathrm{L}$ of $10 \mathrm{X}$ PCR Buffer, $2.5 \mu \mathrm{L}$ of $10 \mathrm{mM}$ dNTPs, $1.5 \mu \mathrm{L}$ of $25 \mathrm{mM} \mathrm{MgCl}_{2}, 3.7 \mu \mathrm{L}$ of primary PCR product, and water $11.8 \mu \mathrm{L}$. The secondary amplification was performed at the following conditions: $94^{\circ} \mathrm{C}, 3$ minutes for initial denaturation, $94{ }^{\circ} \mathrm{C}, 30$ seconds for secondary denaturation and 20 cycles consisting of $47^{\circ} \mathrm{C}, 30$ seconds annealing, $64^{\circ} \mathrm{C}$ for 30 seconds elongation followed by $64^{\circ} \mathrm{C}$ for 3 minutes of final elongation and held at $4{ }^{\circ} \mathrm{C}$. The samples were run in duplicate with one set containing primers targeting the wild type allele (K76) while the second had primers specific for the mutant allele (76T). $5 \mu \mathrm{L}$ of the amplicon was mixed with $5 \mu \mathrm{L}$ of gel loading dye on a parafilm and run concurrently with reference DNA ladder on a $2 \%$ gel. The two products per sample from secondary PCR were run concurrently on agarose gel to discriminate between the wild type, mutant and mixed alleles. Ethidium bromide dye was used to stain the agarose gel for visualization under UV light. The electrophoresis was performed at 97 volts for 30 minutes for both primary and secondary products. The bands on the gels were visualized immediately under UV light system (Ultra Violet Products Inc. Transilluminator). 
217 DNA from W2 and D6 Plasmodium falciparum clones were used as positive controls for mutant 218 and wild type respectively while distilled water was used as a negative control. The negative 219 control was not expected to show any band on gel after electrophoresis while positive control 220 and samples were expected to have sizes of 560 base pairs (bps) for primary product, 342 bps for 221 both mutant and wild type of the secondary product.

Pfmdr1 N86Y and Pfmdr1 Y184F SNP analysis. A fragment of Pfmdr1 gene spanning N86Y

223 and Y184F SNPs was amplified to determine mutations by real-time PCR machine, Applied Biosystems' prism 7500 Fast real-time PCR system (Foster City, CA, USA). The primer and probe sequences used are described in (Purfield et al., 2004). The probes were labeled with 6FAM (for wild type) or VIC (for Mutant), at their 5' ends and non-fluorescent quencher (TAMRA) covalently attached at the 3' end for each. Each well on PCR plate contained both the wild type and mutant type primers and probes. The presence of either or both alleles was showed from the increase of the FAM or VIC fluorophores in distinguishing wild, mutant or mixed alleles. These primers and probes were purchased from Applied Biosystems (Foster City, CA, USA). The reactions were performed in a $25 \mu \mathrm{L}$ reaction mixture made of $18.75 \mu \mathrm{L}$ of the master mix and $6.25 \mu \mathrm{L}$ of the Plasmodium DNA. The master mix consisted; $6.55 \mu \mathrm{L}$ of water, $5 \mu \mathrm{L}$ of $25 \mathrm{mM} \mathrm{MgCl}_{2}, 20 \mu \mathrm{L}$ of Amplitaq Gold, $2.5 \mu \mathrm{L}$ of 10X PCR buffer, $2.5 \mu \mathrm{L}$ of $100 \mathrm{Mm}, 0.5 \mu \mathrm{L}$ of $100 \mu \mathrm{M}$ of Pfmdrl (86 or 184,) forward primer, $0.5 \mu \mathrm{L}$ of $100 \mu \mathrm{M}$ of Pfmdrl (86 or 184) reverse primer, $0.25 \mu \mathrm{L}$ of $100 \mu \mathrm{M}$ of Pfmdrl (86 or 184) wild type probe and $0.25 \mu \mathrm{L}$ of 100 $\mu \mathrm{M}$ of Pfmdrl (86 or 184) mutant probe. Amplification was performed at the following conditions: 1 cycle at $50^{\circ} \mathrm{C}$ for 1 minute, 1 cycle at $95^{\circ} \mathrm{C}$ for 10 minutes and 45 cycles consisting of $95^{\circ} \mathrm{C}$ for 15 seconds followed by $58^{\circ} \mathrm{C}$ for 1 minute.

Quality control was done by running samples and controls in triplicates. Additionally, quality control amplification results were valid only when the positive control (clones W2-mutant and D6-wild type) generated the acceptable average cycle threshold $\left(\mathrm{C}_{\mathrm{T}}\right)$ value $\leq 30$ while the negative control (distilled water) remained unamplified therefore undetected.

Data management and analysis: Numerical data were expressed as proportions and compared using Pearson's Chi-square. Parasitemia expressed as parasites/microliter were log transformed to the natural log and presented as medians with inter quartile range (IQR) and range. Comparison of parasitemia across age and regions was determined by Kruskal-Wallis test by ranks or one-way analysis variance (ANOVA) on ranks. Dunn's pairwise multiple comparison 
248 test was used for post hoc analysis. Univariate and multivariate Logistic regression was used to 249 assess the association of demographic and clinical factors to chloroquine resistance molecular 250 markers. Significance levels were set at 0.05 at 95\% confidence interval. Statistical analyses 251 were performed using Stata version 13 (Stata Corp LP, College Station, Texas) while mapping 252 was done in ArcGIS version 10.

\section{Results}

254 A total of 1,776 subjects presenting with uncomplicated malaria from four malaria epidemic 255 zones across Kenya between 2008 and 2014 were evaluated. The lake endemic zone enrolled the 256 highest number of subjects owing to high disease endemicity followed by highland epidemic 257 zone while Semi-arid seasonal had the least. Their demographic and clinical presentation 258 characteristics are summarized in table 1. Both genders were equally represented with $48.8 \%$ (n $259=867)$ male and 51.2\% $(\mathrm{n}=909)$ female. Majority 56.7\% $(\mathrm{n}=999)$ were children below five years 260 followed by $6-15$ years $21.9 \%(n=386)$ and above 16 years $21.3 \%(n=376)$.

261 All subjects enrolled by this study had a mean temperature of $38.1 \pm 1.21$, mean \pm standard 262 deviation (SD). The chief complaint was fever followed by headache that is in line with classical 263 malaria presentation. At least $62.4 \%(n=1,109)$ subjects had not travelled from their residence 264 within two months prior to enrolment to the study. $80.2 \%(n=1,422)$ reported to have had at least 265 one episode of malaria before date of enrollment. Of these, $10.6 \%(n=188)$ had been treated for 266 malaria in the last 6 weeks prior to enrollment.

\section{Malaria parasite density}

268 The log transformed parasitemia for the 1,776 samples assessed as number of parasites 269 diagnosed by microscopy per microliter of whole blood was median 10.4, IQR 9.1-11.5 and 270 range 3.7-14.2. Using Kruskal-Wallis test by ranks, there was a significant difference [F (3) = $271282.4, p<0.0001]$ in parasitemia across the seven study sites located in the four malaria 272 endemicity zones of Kenya. The lake endemic region had the highest parasitemia median 10.8 273 IQR 9.9-11.6 range 3.7-14.2 followed by coast endemic region median 9.9 IQR 8.8-10.7 range 274 4.8-11.7 while highland epidemic had the least median 8.9, IQR 7.7-10.4, range 4.4-14.2 (Fig. 275 2). Post hoc analysis by Dunn's test showed that the lake endemic region has a significantly 276 higher parasitemia than the rest of the transmission regions $(p<0.05)$ as shown in figure 2. 
277 When comparing parasite burden across age groups, individuals in age group $<5$ years had 278 marginally higher parasitemia median 10.6, IQR 9.3-11.6 range 3.7-13.9 followed by that of age 279 group 6-15 years, median 10.4, IQR 9.2-11.4 range 4.4-14.2. There was a significant difference $280[\mathrm{~F}(2)=54.94, p<0.0001]$ in parasite density among the age groups. Age group $\geq 16$ years 281 parasitemia differed significantly from those of both age groups of $<5$ years and 6-15 years $(p<$ $2820.05)$ (Fig. 3).

\section{Trends of Pfcrt 76, Pfmdr1 86 and Pfm 181184 polymorphisms}

284 We established trends and distribution of chloroquine resistant genotypes; Pfcrt 76, Pfmdr1 86 285 and Pfmdr1 184 across Kenya between 2008 and 2014. The average frequency of the mutant

286

287

288

289

290

291

292

293

294

295

296

297

298

299

300

301

302

303

304

305

306

307 genotype, Pfcrt 76T was 28.3\% (n=446), Wild-type Pfcrt K76 was 49.4\% (n=780) while mixed infection comprising both wild-type and mutant accounted for $22.3 \%(n=353)$ infections countrywide (Table 2). Notably, there was a decline in frequency of Pfcrt 76T single nucleotide polymorphism (SNP) between 2008 and 2014 by $66 \%(\mathrm{n}=125)$ to $5.5 \%(\mathrm{n}=27)$ respectively (Fig. 4). There was a significant difference among the Pfcrt 76 polymorphisms across the years by Chi-square test $\left[\chi^{2}(6)=39.55, p<0.0001\right]$.

The distribution by the current years in the study (2012 to 2014) mutant genotype Pfcrt 76T by malaria endemicity zones showed that Coast endemic region had the highest $34.8 \%(n=8)$ frequency as compared to highland epidemic $11.2 \%(\mathrm{n}=36)$, Lake endemic $26.2 \%(\mathrm{n}=39)$ and semi-arid seasonal with $5.0 \%(n=3)$ as shown in figure 5 . There was a significant difference in frequency of Pfcrt 76T polymorphism across the zones $(p<0.0001)$ across the study period. Distribution by age was $27.6 \%(n=243), 26.9 \%(n=94), 29.9 \%(n=100)$ for ages $\leq 5,6-15, \geq$ 16 years respectively during the study period. There was no difference in Pfcrt 76T frequency across the age groups.

The average frequency of the mutant genotype, $P f m d r 1184 \mathrm{~F}$ was $32.3 \%(\mathrm{n}=507)$, wild type Pfmdr1 Y184 was 47.1\% $(\mathrm{n}=739)$ while mixed infection accounted for $20.6 \%(\mathrm{n}=324)$ infections countrywide (Table 2). A three-fold increase in Pfmdr1 184F, SNP was noted between 2008 and 2014 with frequency of $13.7 \%(n=27)$ to $37.2 \%(n=191)($ Fig. 4). There was a significant difference among the $P f m d r 1$ Y184F polymorphisms across the years $\left[\chi^{2}(6)=17.07\right.$, $p<0.009]$.

The distribution by the current years in the study (2012 to 2014) of mutant genotype Pfmdr1 $184 \mathrm{~F}$ by malaria transmission zones showed a marginally higher frequency in the Semi-arid 
308 seasonal $45.0 \%(n=9)$ followed by Lake endemic 38.4\% $(n=161)$, Highland epidemic 36.8\% (n $309=118)$ and Coast endemic $13.0 \%(\underline{n=3})$ as shown in figure 5. There was a significant difference 310 in frequency of Pfmdr1 184F SNP among the zones $\left(\chi^{2}=17.1, p<0.009\right)$, (Table 2) across the 311 study period. The Pfmdr1 184F, SNP was comparable across all age groups $31.4 \%(\mathrm{n}=280)$

312 during the study period, $34.3 \%(n=118), 32.9 \%(n=105)$ for ages $\leq 5,6-15, \geq 16$ years

313 respectively. There was no difference in $P f m d r 1$ 184F genotype mutation across the age groups.

314 The average frequency of the mutant genotype, $P f m d r 186 \mathrm{Y}$ was $13.7 \%(\mathrm{n}=229)$, wild type

$315 P f m d r 1$ N86 was $73.9 \%(\mathrm{n}=1238)$ while mixed infection comprising both wild-type and mutant 316 accounted for $12.5 \%(\mathrm{n}=209)$ infections countrywide (Table 2). Notably, there was a decline of 317 the Pfmdr1 86Y SNP from 41.8\% $(\mathrm{n}=81)$ to $1.4 \%(\mathrm{n}=7)$ between 2008 and 2014 countrywide 318 (Fig. 4). There was a significant difference among the Pfmdr1 N86Y polymorphism across the 319 years $\left[\chi^{2}(6)=21.87, p<0.001\right]$.

320 The distribution by current years in the study (2012 to 2014) of mutant genotype, $P f m d r 186 \mathrm{Y}$ by 321 malaria transmission zones showed that the coastal endemic had the highest $30.4 \%(n=7)$ 322 frequency followed by Semi-arid seasonal 10\% $(\mathrm{n}=2)$, Lake endemic 3.3\% $(\mathrm{n}=14)$ and 323 Highland epidemic 2.2\% $(\mathrm{n}=7)$ (Fig.5). There was a significant difference in frequency of

$324 P f m d r 1$ 86Y SNP (Table 2) across the study period and was comparable among all age groups $32512.8 \%(\mathrm{n}=122), 13.4 \%(\mathrm{n}=49), 16.3 \%(\mathrm{n}=56)$ for ages $\leq 5,6-15, \geq 16$ years respectively 326 during the study period. There was no difference in $P f m d r 186$ genotype mutation across the age 327 groups.

\section{Demographic and clinical factors associated with chloroquine resistance}

329 To explore the association between demographic factors and chloroquine resistance (CQR) based 330 on Pfcrt 76 allele, a CQR primary marker, univariate and multivariate logistic regression was used (Table 3). Demographic factors included sex, age group, and travel in the last 2 months, ever had malaria, treated for malaria within the last 6 weeks and the malaria transmission zones. Sex was significantly associated with CQR, $p<0.027$ by univariate model and $p<0.025$ by multivariate model. Males were more likely to have CQR, 1.28 times by univariate model and 1.29 times by multivariate model. On the other hand, age was not significantly associated with CQR, though it's important to note that subjects above 6 years of age were about one more time likely to harbor resistant genotypes. There was a significant relationship between having 
339 model $(p<0.002)$, with subjects who had travelled being one or more time likely to be

340 chloroquine resistant than those who had not. Having had malaria infection prior to the present

341 visit was not associated with CQR, but individuals who had suffered from malaria before were

342 one more time likely to be CQR than those who had no malaria infection prior to study

343 enrollment. On the contrary, treatment in the last 6 weeks before visit was significantly

344 associated with CQR by multivariate model $(p<0.034)$. Individuals who had treatment six

345 weeks prior to the visit were one more time likely to acquire CQR. No association between

346 living in malaria epidemic zones and CQR was observed. However, individuals in Lake endemic

347 region are more likely to be CQR by 1.52 times (univariate model) and 1.87 times (multivariate

348 model). Lake endemic region was followed by highland epidemic while people living in semiarid

349 seasonal were less likely to be CQR.

350

351

\section{Discussion}

352 Antimalarial resistance has significantly hindered malaria control efforts. Since effective

353 vaccines for malaria are still way off, and drugs are most relied upon, success of malaria control

354 strategies are dependent on understanding factors that influence dispersion of malaria drug

355 resistance genotypes. Most studies on CQR appear inconclusive on methods of containing it's

356 spread due to insufficient demographic and clinical information (Akala et al., 2014; Angira,

357 Otieno, Muga, \& Abong'o, 2010; Zhong et al., 2008). This study showed that travel, gender and

358 clinical factors were associated with chloroquine resistance among symptomatic malaria cases in

359 four of the five malaria epidemic zones in Kenya. Additionally, this study established that while

360 Pfcrt 76 and $P f m d r 186$ SNPs had reduced, the $P f m d r 1184$ SNP was on the rise.

361 Travel and gender were significantly associated with CQR among symptomatic malaria

362 individuals by both univariate and multivariate model (Table 3 ). The association between gender

363 and CQR implies that males are more likely to be chloroquine resistant than females, though

364 literature indicates that gender norms exposes females and children to the risk of malaria

365 (Ministry of Health, 2015). On the contrary, recent studies in Yemen and Malaysia did not show

366 association between CQR and gender (Atroosh, Al-Mekhlafi, Mahdy, \& Surin, 2012; Bamaga,

367 Mahdy, \& Lim, 2015) despite compelling evidence of potential role of gender in epidemiology

368 of malaria elsewhere (Diiro et al., 2016; WHO, 2007). The contrary findings could be attributed

369 to the social cultural values around gender. A study done on prevalence of asymptomatic and 
370 submicroscopic malaria infections on Islands in Lake Victoria, Kenya, reported to have a

371 significant prevalence of malaria in males than in females $(p<0.005)$ (Idris et al., 2016).

372 Globally, gender dimensions also influence access to treatment, care for malaria and use of

373 preventive measures (WHO, 2007). Females play the primary role of care giving to other

374 members in the household, including leading the majority of health care seeking role for the rest

375 of the family members (Diiro et al., 2016; Roll back malaria partnership, 2016) though males

376 still dominate decision making on health and economic issues in the households (Diiro et al.,

377 2016). Malaria is "gender blind" as mosquitoes bite indiscriminately (Ministry of Health, 2015).

378 However, in endemic regions, males may delay seeking treatment and risk accumulating super

379 infection. Research has shown that mutant strains that develop drug resistance enhance virulence

380 (Shahinas, Folefoc, \& Pillai, 2013) wanton utilization of resources and therefore eliciting

381 symptoms that distorts the host/parasite balance that would allow wild type parasites to remain

382 asymptomatic (Akala et al., 2014; Deroost, Pham, Opdenakker, \& Van den Steen, 2016).

383 The current finding on association of travel and CQR is concurrent with similar studies reported.

384 A study done in Ethiopia reported effects of migration on parasite gene flow promoting 385 transmission of chloroquine resistance (Lo et al., 2017). At a smaller scale, another study in

386 Congo, reported evidence of imported resistant $P$. falciparum strains into Guatemala from Congo

387 given globalization and international travels. Travelers from endemic regions with malaria

388 symptoms should be suspected of harboring chloroquine resistant strains (Patel et al., 2014)

389 Human population movement has been shown to contribute to spread of drug resistant parasite

390 strains elsewhere (Lynch \& Roper, 2011).

391 It was also noteworthy that treatment in the last 6 weeks before enrollment was not associated

392 with CQR by univariate model but was associated by multivariate model. This is an implication

393 that other factors contribute to CQR. Literature shows that irrational use of drugs (Nsubuga et

394 al., 2011) gender dimensions (WHO, 2007), travel (Juliao et al., 2013), number of parasites

395 exposed to a drug, the drug concentration to which the parasites are exposed, and the

396 simultaneous presence of other antimalarials in the blood to which the parasite is not resistant

397 influence the emergence and spread of drug resistant malaria parasites (WHO, 2018). These

398 multiple factors argue for deployment of integrated research for effective malaria control. Such a

399 strategy would include systems thinking, out-come-oriented monitoring and evaluation 
400 approaches, collaboration - across disciplines, sectors and regions -, multi-stakeholder

401 engagement, and sensitivity to social equity (Wiese, 2012).

402 Other factors such as age, ever had malaria and location by epidemic zone were not associated

403 with CQR. However, age has been shown to be a risk factor of malaria with a vast majority of

404 malaria cases occurring under age of 5 years (WHO, 2016b). Though children who live in

405 endemic areas may have parasites with drug resistance to Plasmodium falciparum, they often

406 recover after chemotherapy. This is an indication that acquired immunity works in synergy with

407 antimalarials (Enevold et al., 2007). On the other hand, the history of malaria is important in

408 determining recurrent malaria, recrudescence of malaria, relapse and infection (Velho, Alves-jr,

409 Ribatski-silva, \& Gomes, 2014). Recurrence has been reported to be due to one or many of the

410 following: therapeutic failure resulting from non-adherence to treatment, resistance of the

411 parasite to the drug-use, poor quality of the medication, or sub-therapeutic doses of the drugs; (b)

412 reactivation of hypnozoites; and (c) exposure to new infection by the mosquito vector (Hedt,

413 Laufer, \& Cohen, 2011; White, 2011). Additionally, though location by epidemic zones was also

414 not associated with $\mathrm{CQR}$, determining location of origin of patients who present with malaria is

415 key for successful intervention strategies (Pindolia et al., 2013).

416 High parasitemia was noted for samples from across all sites (Fig.2) and age groups (Fig.3) in

417 Kenya regardless of the difference in disease transmission burden. Studies suggest that residents

418 of holoendemic regions are often immune to malaria (Rolfes et al., 2012). They therefore can

419 harbor parasitemia for long prior to showing symptoms than those in non-immune individuals

420 residing in highland epidemic and semi-arid seasonal transmission zones pf Kenya. It is however

421 worth noting that microscopy that is the Kenya ministry of health's guidelines recommended

422 diagnosis method is less sensitive for detection of low parasitemia (Lo et al., 2015), a factor that

423 could have excluded individuals with low parasitemia. Additionally, a study done in Tanzania

424 reported a two-fold difference in parasite density when samples were collected at two time points

425 (-2 and 0 hours) prior to treatment (Carlsson et al., 2011), It is therefore possible that parasitemia

426 for low transmission region would be higher than that reported here if all sub-microscopic

427 parasitemia's were depicted using sensitive methods and if two samples were collected at

428 different time points. Further, the study design that included only symptomatic individuals with

429 detectable malaria could have been biased towards individuals with higher load across all sites 
430 though similar studies in Southeast Asia have reported significantly lower parasitemia

431 (Chaorattanakawee et al., 2013).

432 Analysis of trends and patterns of distribution of chloroquine resistant genotypes revealed a 433 notable decline by $56.7 \%$ in the Pfcrt $76 \mathrm{~T}$ and $38.8 \%$ for the Pfmdr $186 \mathrm{Y}$ mutant genotypes 434 between 2008 and 2014 (Fig.4),. This presents a possibility for future introduction of chloroquine 435 use for malaria treatment as a combination therapy. On the other hand, this observation indicates 436 an increased risk of reinfection after treatment with AL. A study done to determine effects of $437 P f c r t$ and $P f m d r 1$ gene polymorphisms on therapeutic responses to artesunate-amodiaquine 438 (ASAQ) and AL reported that parasites with the Pfmdr 1 N86, and $P f c r t \mathrm{~K} 76$ alleles re-infected 439 patients earlier after AL treatment (Venkatesan et al., 2014), due to selection of less susceptible 440 parasites (Sisowath et al., 2009). Another study done on genetically engineered P. falciparum 441 lines reported that Pfmdrl N86Y mutation increased parasite susceptibility to a wide range of 442 first line antimalarials including Lumefantrine and Dihydroartemisinin (DHA) while decreasing 443 parasite susceptibility to CQ. Pfmdr1 N86Y mutation was reported to alter parasite response 444 DHA when compared to N86 parasites (Veiga et al., 2016). The mixed genotype Pfcrt K76T 445 mutant and wild type allele is directly linked to both in vitro and clinical resistance. Therefore, it 446 can still be used as a biomarker for CQ resistance (Wellems \& Plowe, 2001).

447 On the other hand, there was a reciprocal increase for the Pfmdr 1 184F mutant genotype from $448 \quad 13.7 \%$ to $37.2 \%$ representing a $23.5 \%$ rise between 2008 and 2014 (Fig.4). The implication of 449 Pfm $d r 1$ Y184F mutation has been reported to be minimal on antimalarial drug susceptibilities 450 (Veiga et al., 2016). This observation was contrary to a study reported in Grande Comore Island, 451 Comoros between 2006-2014 where Pfmdr1 184F mutations dropped from 52.2 to $30.0 \%$ 452 ( $\mathrm{p}<0.01)$ (Huang et al., 2016). Another study done in Guinea-Bissau between 2003 and 2012 453 reported that there was no significant change in the Pfmdr 1 184F mutant allele (Jovel, Kofoed, 454 Rombo, Rodrigues, \& Ursing, 2015). A study done in Yemen, Tehama region reported a high 455 prevalence of $P f m d r 1$ 184F mutant allele (99\%) (Atroosh et al., 2016)and an increase from 19.6 456 to $22.7 \%$ in 2010 -2012 in Maputo after introduction of ACT (Lobo et al., 2014). In Kenya, a 457 significant association between polymorphisms at the Pfmdr1 184 and lumefantrine have been 458 reported (Achieng et al., 2015). However, a previous study from Odisha has reported the high 459 prevalence of $P f m d r 186 \mathrm{Y}$ mutation and low prevalence of $P f m d r 1$ 184F mutation (Antony, Das, 460 Parija, \& Padhi, 2016). Additionally, increased Pfmdrl copy number has been associated with 
461 treatment failure after short 4-dose AL therapy (Sisowath et al., 2009). The increased Pfmdr1 462 copy numbers has been reported to be a rare observation in African population due to little 463 exposure to MQ (Venkatesan et al., 2014).

464 Significant variation in genotype distribution was noted; the Coast and Lake endemic regions 465 had the highest frequency of $31.6 \%$ and $29.6 \%$ for the $P$ fcrt $76 \mathrm{~T}$ mutant allele respectively 466 (Table 2). Genotype distribution for year 2014 (Fig.5) by spatial analysis also showed varied 467 distribution (Fig.5). This observation could be partly due to inadequate enforcement on the ban 468 of CQ and that CQ is still in use after its official withdrawal in Kenya (Rebelo, Tempera, 469 Fernandes, Grobusch, \& Hänscheid, 2015). The coastal region had the highest Pfmdr1 86Y 470 mutant allele with 23.4\%. CQ selects for parasites with Pfmdr $186 \mathrm{Y}$ mutation (Li et al., 2014). 471 Semi-Arid seasonal had the highest Pfmdr 1 184F mutant allele with $32.5 \%$ while the coastal 472 endemic region had the highest Pfmdr 1 Y 184 allele at $57.8 \%$ suggesting that the population in 473 the Semi-Arid seasonal zone have a higher risk to parasite tolerance to some antimalarials. It has 474 been reported that polymorphism in the $P f m d r 1$ gene is associated with an increase in parasite 475 tolerance/resistance to some anti-malarials (Atroosh et al., 2016). This would also imply 476 increased use of lumefantrine in the region. It has been reported that existence of $P f m d r 1184 \mathrm{~F}$ 477 mutant allele increases with the use of lumefantrine (Malmberg et al., 2013; Sisowath et al., 478 2007). An increase of $18.4 \%$ to $23.5 \%$ access to AL from within 48 hours of fever on set had 479 been reported in 2015 (Wasunna et al., 2015), after implementation of a new drug policy in 480 Kenya 2004 (Gitonga et al., 2008; Watsierah, Onyango, Ombaka, Abong 'o, \& Ouma, 2012).

481 The high parasitemia means that individuals living in the Lake endemic region will consequently 482 have high rates of transmission of $\mathrm{CQR}$ genotypes as compared to other regions.

483 Previous studies show increasing chloroquine sensitivity (Eyase et al., 2013; Kiarie, Wangai, 484 Agola, Kimani, \& Hungu, 2015; Mohammed et al., 2013) after it was replaced by sulfadoxine485 pyrimethamine in 1998 (Eyase et al., 2013). A study done in Tanzania reported $>90 \%$ recovery 486 of CQ susceptibility ten years after its withdrawal (Mohammed et al., 2013). Two studies done 487 in Western Kenya reported a significant difference to CQ sensitivity in the Pfcrt 76 codon 488 between 2008 and 2011 p $<0.001$ (Eyase et al., 2013), and a decline from 76\% to 6\% of Pfcrt 489 76T prevalence from 2003 to 2015 (Vardo-Zalik et al., 2018). Another study done in Msabweni 490 Kenya reported a 41\% (n-99) in the Pfcrt 76T mutant allele in the year 2013 representing a 491 significant decline in frequency compared to $2006(\mathrm{p} \leq 0.05)$ (Kiarie et al., 2015). Similarly, this 
492 study shows increasing frequency of $P f c r t \mathrm{~K} 76$ and $P f m d r 1$ N86 genotypes that are suggestive of 493 increasing CQ sensitivity in the four out of five malaria endemicity zones of Kenya heralding 494 possible reintroduction of CQ based combination treatment. On the other hand, the prevalence 495 Pfm $d r 1184 \mathrm{~F}$ genotype increased during the 2008-14 period. The Pfmdr 1 184F mutation has 496 been associated with AL resistance (Antony et al., 2016), and poses a threat to the sensitivity of 497 lumefantrine and MQ (Ministry of Health, 2010). Lumefantrine in combination with artemether 498 has been the recommended first-line treatment for uncomplicated malaria in Kenya since 2006 499 (Gitonga et al., 2008; Lucchi et al., 2015; Ministry of Health, 2010), while MQ is used as a 500 chemoprophylaxis in Kenya (Ministry of Health, 2010).

501 Studies have shown that understanding the patterns of parasite dispersal from local hotspots of 502 transmission can aid the design of additional targeted control by identifying both the regions 503 where imported infections originate and where they may contribute substantially to transmission 504 (Wesolowski et al., 2012). It will therefore be of great importance to establish gene flow 505 patterns of Plasmodium falciparum resistant genotypes within the Kenyan population. This will 506 be crucial in designing strategies towards control and prevention of malaria per epidemiological 507 zone.

508 The limitation to this study was using a convenience sample. This implies that the sample size 509 used in some regions could not be a representative of the whole population. Univariate and 510 multivariate regression was used to analyze data that was stratified by age, gender and location to 511 ensure that the analysis outcome is not due to confounding factors, bias or effect modifiers.

\section{Conclusions}

513 According to this study gender and travel have contributed to the dispersion of CQR genotypes

514 in Kenya. The coast and lake endemic region bears the brunt of the burden among other regions. 515 Additionally, children under the age of 5 years remain to be at a higher risk of malaria burden. 516 Decline in CQR based on Pfcrt 76T SNP, is an indication that CQ can be re-considered for 517 treatment of $P f$ malaria in future. An increase in $P f m d r 1 \mathrm{~N} 86$ wild type implies tolerance to 518 lumefantrine. On the other hand, increased $P f m d r 1$ 184F mutant genotype frequency indicates a 519 reduced sensitivity to lumefantrine. This study recommends continuous monitoring of CQR with 520 wholesome information. Further studies should be done to determine migration patterns of $P f$ 
521 CQ resistant genotypes and gender factors associated with CQR in the malaria epidemic zones in 522 Kenya.

\section{Acknowledgements}

524 We thank all GEIS clinical staff serving at the sentinel sites for their assistance and the study

525 subjects and their parents/guardians who participated in the study and contributed their samples 526 for study.

527

\section{References}

529 Achieng, A. O., Muiruri, P., Ingasia, L. A., Opot, B. H., Juma, D. W., Yeda, R., ... Kamau, E.

530 (2015). International Journal for Parasitology : Drugs and Drug Resistance Temporal trends

531

532 in prevalence of Plasmodium falciparum molecular markers selected for by artemether-

533 lumefantrine treatment in pre- ACT and post-ACT parasites in western Kenya. International

534 Journal for Parasitology: Drugs and Drug Resistance, 5(3), 92-99. https://doi.org/10.1016/j.ijpddr.2015.05.005

535

Afrane, Y., Zhou, G., Githeko, A., \& Yan, G. (2014). Clinical malaria case definition and

536

537 malaria attributable fraction in the highlands of western Kenya. 1-7.

Akala, H. M., Achieng, A. O., Eyase, F. L., Juma, D. W., Ingasia, L., Cheruiyot, A. C., ...

538

539

540

541

Amambua-Ngwa, A., Amenga-Etego, L., Kamau, E., Amato, R., Ghansah, A., Golassa, L., ...

542

543

544 Kamau, E. (2014). Five-year tracking of plasmodium falciparum allele frequencies in a holoendemic area with indistinct seasonal transitions. Journal of Multidisciplinary

545

Angira, C. H. O., Otieno, O. A., Muga, R. O., \& Abong’o, B. O. (2010). Factors contributing to antimalarial drug resistance in Rachuonyo district, Kenya. East African Journal of Public Health, 7(1), 11-15. Retrieved from http:/www.ncbi.nlm.nih.gov/pubmed/21413566 genes and its association with chloroquine resistance in Southeast Indian Plasmodium falciparum isolates. Genomics Data, 8(2016), 85-90. 
551

552

553

554

555

556

557

558

559

560

561

562

563

564

565

566

567

568

569

570

571

572

573

574

575

576

577

578

579

580

581

https://doi.org/10.1016/j.gdata.2016.04.010

Aribodor D.N, Ugwuanyi I.K., \& Aribodor O.B. (2016). Challenges to Achieving Malaria Elimination in Nigeria. American Journal of Public Health Research, 4(1), 38-41. https://doi.org/10.12691/ajphr-4-1-6

Atroosh, W. M., Al-Mekhlafi, H. M., Al-Jasari, A., Sady, H., Dawaki, S. S., Elyana, F. N., ... Surin, J. (2016). Different patterns of $p f c r t$ and $p f m d r 1$ polymorphism in Plasmodium falciparum isolates from Tehama region, Yemen. PeerJ, 4, e2191. https://doi.org/10.7717/peerj.2191

Atroosh, W. M., Al-Mekhlafi, H. M., Mahdy, M. A., \& Surin, J. (2012). The detection of pfcrt and pfmdr1 point mutations as molecular markers of chloroquine drug resistance, Pahang, Malaysia. Malaria Journal, 11(1), 251. https://doi.org/10.1186/1475-2875-11-251

Bamaga, O. A. A., Mahdy, M. A. ., \& Lim, Y. A. . (2015). Survey of chloroquine-resistant mutations in the Plasmodium falciparum pfcrt and pfmdr-1 genes in Hadhramout, Yemen. Acta Tropica, 149, 59-63. https://doi.org/10.1016/J.ACTATROPICA.2015.05.013

Bloland, P. B. (2001). WHO/CDS/CSR/DRS/2001.4 Drug resistance in malaria. Retrieved from http://www.who.int/emc

Borrmann, S., Matsiegui, P. B., Missinou, M. A., \& Kremsner, P. G. (2008). Effects of Plasmodium falciparum parasite population size and patient age on early and late parasitological outcomes of antimalarial treatment in children. Antimicrobial Agents and Chemotherapy, 52(5), 1799-1805. https://doi.org/10.1128/AAC.00755-07

Carlsson, A. M., Ngasala, B. E., Dahlström, S., Membi, C., Veiga, I. M., Rombo, L., ... Mårtensson, A. (2011). Plasmodium falciparum population dynamics during the early phase of anti-malarial drug treatment in Tanzanian children with acute uncomplicated malaria. Malaria Journal, 10. https://doi.org/10.1186/1475-2875-10-380

Chaorattanakawee, S., Tyner, S. D., Lon, C., Yingyuen, K., Ruttvisutinunt, W., Sundrakes, S., ... Lanteri, C. A. (2013). Direct comparison of the histidine-rich protein-2 enzyme-linked immunosorbent assay (HRP-2 ELISA) and malaria SYBR green i fluorescence (MSF) drug sensitivity tests in Plasmodium falciparum reference clones and fresh ex vivo field isolates from Cambodia. Malaria Journal, 12(1). https://doi.org/10.1186/1475-2875-12-239

Cohen, J. M., Smith, D. L., Cotter, C., Ward, A., Yamey, G., Sabot, O. J., \& Moonen, B. (2012). Malaria resurgence: a systematic review and assessment of its causes. Malaria Journal, 
582

583

584

585

586

587

588

589

590

591

592

593

594

595

596

597

598

599

600

601

602

603

604

605

606

607

608

609

610

611

612

11(1), 122. https://doi.org/10.1186/1475-2875-11-122

Deroost, K., Pham, T. T., Opdenakker, G., \& Van den Steen, P. E. (2016). The immunological balance between host and parasite in malaria. FEMS Microbiology Reviews, 40(2), 208257. https://doi.org/10.1093/femsre/fuv046

Desai, M., Gutman, J., L’lanziva, A., Otieno, K., Juma, E., Kariuki, S., ... ter Kuile, F. (2015). Intermittent screening and treatment (IST) or 4 intermittent preventive treatment (IPT) with dihydroartemisinin-piperaquine versus IPT with 5 sulphadoxine-pyrimethamine for the control of malaria in pregnancy in western Kenya: A 6 randomized controlled su. Lancet Global Health, 3(15), 712-723. https://doi.org/10.1016/S0140-6736(15)01274-X

Dhiman, S. (2019, February 13). Are malaria elimination efforts on right track? An analysis of gains achieved and challenges ahead. Infectious Diseases of Poverty, Vol. 8. https://doi.org/10.1186/s40249-019-0524-X

Diiro, G. M., Affognon, H. D., Muriithi, B. W., Wanja, S. K., Mbogo, C., Mutero, C., ... Aliber, M. (2016). The role of gender on malaria preventive behaviour among rural households in Kenya. Malaria Journal, 15(1), 14. https://doi.org/10.1186/s12936-015-1039-y

Enevold, A., Nkya, W. M., Theisen, M., Vestergaard, L. S., Jensen, A. T., Staalsoe, T., ... Druilhe, P. (2007). Potential impact of host immunity on malaria treatment outcome in Tanzanian children infected with Plasmodium falciparum. Malaria Journal, 6(1), 153. https://doi.org/10.1186/1475-2875-6-153

Eyase, F. L., Akala, H. M., Ingasia, L., Cheruiyot, A., Omondi, A., Okudo, C., ... Johnson, J. D. (2013). The Role of Pfmdr1 and Pfcrt in Changing Chloroquine, Amodiaquine, Mefloquine and Lumefantrine Susceptibility in Western-Kenya P . falciparum Samples during 2008 - 2011. PloS One, 8(5). https://doi.org/10.1371/journal.pone.0064299

Gitonga, C. W., Amin, A. A., Ajanga, A., Kangwana, B. B., Noor, A. M., \& Snow, R. W. (2008). The use of artemether-lumefantrine by febrile children following national implementation of a revised drug policy in Kenya. Tropical Medicine \& International Health : TM \& IH, 13(4), 487-494. https://doi.org/10.1111/j.1365-3156.2008.02026.x

Gyasi, A., Asante, K., Newton, S., Amoako, S., Dosoo, D., Ankrah, L., ... S., O.-A. (2015). Malaria parasite density estimated with white blood cells count reference value agrees with density estimated with absolute in children less than 5 years in central Ghana. Malaria Research and Treatment, 1155(10). https://doi.org/10.1155/2015/923674 
613 Hastings, I. M., \& Donnelly, M. J. (2005). The impact of antimalarial drug resistance mutations 614 on parasite fitness, and its implications for the evolution of resistance. Drug Resistance 615 Updates, 8(1-2), 43-50. https://doi.org/10.1016/j.drup.2005.03.003

616 Hedt, B. L., Laufer, M. K., \& Cohen, T. (2011). Drug resistance surveillance in resource-poor 617 settings: Current methods and considerations for TB, HIV, and malaria. American Journal 618 of Tropical Medicine and Hygiene, 84(2), 192-199. https://doi.org/10.4269/ajtmh.2011.106190363

620 Huang, B., Wang, Q., Deng, C., Wang, J., Yang, T., Huang, S., ... Song, J. (2016). Prevalence of 621 crt and mdr-1 mutations in Plasmodium falciparum isolates from Grande Comore island 622 after withdrawal of chloroquine. Malaria Journal, 15(1), 414.

623 https://doi.org/10.1186/s12936-016-1474-4

624 Idris, Z., Chan, C. W., Kongere, J., Gitaka, J., Logedi, J., Omar, A., ... Kaneko, A. (2016). High 625 and Heterogeneous Prevalence of Asymptomatic and Sub-microscopic Malaria Infections 626 on Islands in Lake Victoria, Kenya. Scientific Reports, 6(1), 36958.

627 https://doi.org/10.1038/srep36958

628 Jovel, I. T., Kofoed, P. E., Rombo, L., Rodrigues, A., \& Ursing, J. (2015). Temporal and 629 seasonal changes of genetic polymorphisms associated with altered drug susceptibility to 630 chloroquine, lumefantrine, and quinine in Guinea-Bissau between 2003 and 2012.

631 Antimicrobial Agents and Chemotherapy, 59(2), 872-879.

632 https://doi.org/10.1128/AAC.03554-14

633 Juliao, P. C., Sosa, S., Gonzalez, L. D., Padilla, N., Ortiz, L., Goldman, I., ... Lindblade, K. A. 634 (2013). Importation of chloroquine-resistant Plasmodium falciparum by Guatemalan 635 peacekeepers returning from the Democratic Republic of the Congo.

636 https://doi.org/10.1186/1475-2875-12-344

637 Kamau, E., Campino, S., Amenga-Etego, L., Drury, E., Ishengoma, D., Johnson, K., ... Djimde, 638 A. A. (2015). K13-propeller polymorphisms in plasmodium falciparum parasites from sub639 640 saharan Africa. Journal of Infectious Diseases, 211(8), 1352-1355.

641 https://doi.org/10.1093/infdis/jiu608

Kiarie, W. C., Wangai, L., Agola, E., Kimani, F. T., \& Hungu, C. (2015). Chloroquine sensitivity: diminished prevalence of chloroquine-resistant gene marker pfcrt-76 13 years after cessation of chloroquine use in Msambweni, Kenya. Malar J, 14, 328. 
644 https://doi.org/10.1186/s12936-015-0850-9

645 Li, J., Chen, J., Xie, D., Monte-Nguba, S., Eyi, J. U. M., Matesa, R. A., .. Lin, M. (2014). High

646 prevalence of pfmdr1 N86Y and Y184F mutations in Plasmodium falciparum isolates from

647 Bioko island, Equatorial Guinea. Pathogens and Global Health, 108(7), 339-343.

648 https://doi.org/10.1179/2047773214Y.0000000158

649 Lo, E., Hemming-Schroeder, E., Yewhalaw, D., Nguyen, J., Kebede, E., Zemene, E., ... Yan, G.

650 (2017). Transmission dynamics of co-endemic Plasmodium vivax and P. falciparum in

651 Ethiopia and prevalence of antimalarial resistant genotypes. PLOS Neglected Tropical

652 Diseases, 11(7), e0005806. https://doi.org/10.1371/journal.pntd.0005806

653 Lo, E., Zhou, G., Oo, W., Afrane, Y., Githeko, A., \& Yan, G. (2015). Low parasitemia in

654 submicroscopic infections significantly impacts malaria diagnostic sensitivity in the

655 highlands of Western Kenya. PLoS ONE, 10(3), 1-15.

656 https://doi.org/10.1371/journal.pone.0121763

657 Lobo, E., de Sousa, B., Rosa, S., Figueiredo, P., Lobo, L., Pateira, S., .. Nogueira, F. (2014).

658 Prevalence of pfmdr1 alleles associated with artemether-lumefantrine tolerance/resistance in

659 Maputo before and after the implementation of artemisinin-based combination therapy.

660 Malaria Journal, 13(1), 300. https://doi.org/10.1186/1475-2875-13-300

661 Lucchi, N. W., Komino, F., Okoth, S. A., Goldman, I., Onyona, P., Wiegand, R. E., ... Kariuki,

662 S. (2015). In Vitro and Molecular Surveillance for Antimalarial Drug Resistance in

663 Plasmodium falciparum Parasites in Western Kenya Reveals Sustained Artemisinin

664 Sensitivity and Increased Chloroquine Sensitivity. Antimicrobial Agents and Chemotherapy,

665 59(12), 7540-7547. https://doi.org/10.1128/AAC.01894-15.Address

666 Lynch, C., \& Roper, C. (2011). The Transit Phase of Migration: Circulation of Malaria and Its

667 Multidrug-Resistant Forms in Africa. PLoS Medicine, 8(5), e1001040.

668 https://doi.org/10.1371/journal.pmed.1001040

669 Malmberg, M., Ferreira, P. E., Tarning, J., Ursing, J., Ngasala, B., Björkman, A., ... Gil, J. P.

670 (2013). Plasmodium falciparum drug resistance phenotype as assessed by patient

671 antimalarial drug levels and its association with pfmdr1 polymorphisms. Journal of

672 Infectious Diseases, 207(5), 842-847. https://doi.org/10.1093/infdis/jis747

673 Mbugi, E. V, Mutayoba, B. M., Malisa, A. L., Balthazary, S. T., Nyambo, T. B., \& Mshinda, H.

674 (2006). Drug resistance to sulphadoxine-pyrimethamine in Plasmodium falciparum malaria 
675

676

677

678

679

680

681

682

683

684

685

686

687

688

689

690

691

692

693

694

695

696

697

698

699

700

701

702

703

704

705

in Mlimba, Tanzania. Malaria Journal, 5(1), 94. https://doi.org/10.1186/1475-2875-5-94

Ministry of Health. (2010). National Guidelines for the Diagnosis, Treatment and Prevention of Malaria in Kenya. Retrieved from

http://www.thehealthcompass.org/sites/default/files/project_examples/Kenya_Malaria_Tx_ Guideline_2010.pdf

Ministry of Health, K. (2015). Gender and Malaria in Kenya Gender and Malaria in Kenya.

Mohammed, A., Ndaro, A., Kalinga, A., Manjurano, A., Mosha, J. F., Mosha, D. F., ... Kavishe, R. A. (2013). Trends in chloroquine resistance marker, Pfcrt-K76T mutation ten years after chloroquine withdrawal in Tanzania. Malaria Journal, 12(1), 415. https://doi.org/10.1186/1475-2875-12-415

Nosten, F., \& White, N. J. (2007). Artemisinin-Based Combination Treatment of Falciparum Malaria. The American Society of Tropical Medicine and Hygiene, 77(Suppl 6), 181-192.

Nsubuga, P., Johnson, K., Tetteh, C., Oundo, J., Weathers, A., Vaughan, J., ... Njenga, K. (2011). Field epidemiology and laboratory training programs in sub-saharan Africa from 2004 to 2010: Need, the process, and prospects. Pan African Medical Journal, 10(November), 0-12. https://doi.org/10.4314/pamj.v10i0.72235

Patel, J. C., Taylor, S. M., Juliao, P. C., Parobek, C. M., Janko, M., Gonzalez, L. D., ... Meshnick, S. R. (2014). Genetic Evidence of Importation of falciparum to Guatemala from the Democratic Republic of the Congo. Emerging Infectious Diseases, 20(6), 932-940.

Pindolia, D. K., Garcia, A. J., Huang, Z., Smith, D. L., Alegana, V. A., Noor, A. M., ... Tatem, A. J. (2013). The demographics of human and malaria movement and migration patterns in East Africa. Malaria Journal, 12, 397. https://doi.org/10.1186/1475-2875-12-397

Purfield, A., Nelson, A., Laoboonchai, A., Congpuong, K., McDaniel, P., Miller, R. S., ... Snounou, G. (2004). A new method for detection of pfmdr1 mutations in Plasmodium falciparum DNA using real-time PCR. Malaria Journal, 3(1), 9. https://doi.org/10.1186/1475-2875-3-9

Rebelo, M., Tempera, C., Fernandes, J. F., Grobusch, M. P., \& Hänscheid, T. (2015). Assessing anti-malarial drug effects ex vivo using the haemozoin detection assay. Malaria Journal, 14(1), 140. https://doi.org/10.1186/s12936-015-0657-8

Rolfes, M. A., McCarra, M., Magak, N. G., Ernst, K. C., Dent, A. E., Lindblade, K. A., \& John, C. C. (2012). Development of clinical immunity to malaria in highland areas of low and 
706

707

708

709

710

711

712

713

714

715

716

717

718

719

720

721

722

723

724

725

726

727

728

729

730

731

732

733

734

735

736

unstable transmission. American Journal of Tropical Medicine and Hygiene, 87(5), 806812. https://doi.org/10.4269/ajtmh.2012.11-0530

Roll back malaria partnership. (2016). Gender and Malaria (Vol. 667).

Shahinas, D., Folefoc, A., \& Pillai, D. R. (2013). Targeting Plasmodium falciparum Hsp90: Towards Reversing Antimalarial Resistance. Pathogens, 2(1), 33-54. https://doi.org/10.3390/pathogens2010033

Shetty, A. C., Jacob, C. G., Huang, F., Li, Y., Agrawal, S., Saunders, D. L., ... Onyamboko, M. A. (2019). Genomic structure and diversity of Plasmodium falciparum in Southeast Asia reveal recent parasite migration patterns. Nature Communications, 10(1). https://doi.org/10.1038/s41467-019-10121-3

Sisowath, C., Ferreira, P. E., Bustamante, L. Y., Dahlström, S., Mårtensson, A., Björkman, A., ... Gil, J. P. (2007). The role of pfmdr1 in Plasmodium falciparum tolerance to artemetherlumefantrine in Africa. Tropical Medicine and International Health, 12(6), 736-742. https://doi.org/10.1111/j.1365-3156.2007.01843.x

Sisowath, C., Petersen, I., Veiga, M. I., Mårtensson, A., Premji, Z., Björkman, A., ... Gil, J. P. (2009). In Vivo Selection of Plasmodium falciparum Parasites Carrying the ChloroquineSusceptible pfcrt K76 Allele after Treatment with Artemether-Lumefantrine in Africa . Journal of Infectious Diseases, 199(5), 750-757. https://doi.org/10.1086/596738

Vardo-Zalik, A., Dixit, A., Zhong, D., Atieli, H., Umukoro, E., Hemming-Schroeder, E., ... Tomás-Domingo, P. (2018). Impacts of Antimalarial Drugs on Plasmodium falciparum Drug Resistance Markers, Western Kenya, 2003-2015. The American Journal of Tropical Medicine and Hygiene, tpmd170763. https://doi.org/10.4269/ajtmh.17-0763

Veiga, M. I., Dhingra, S. K., Henrich, P. P., Straimer, J., Gnädig, N., Uhlemann, A.-C., ... Fidock, D. A. (2016). Globally prevalent PfMDR1 mutations modulate Plasmodium falciparum susceptibility to artemisinin-based combination therapies. Nature Communications, 7, 11553. https://doi.org/10.1038/ncomms11553

Velho, D. P., Alves-jr, E. R., Ribatski-silva, D., \& Gomes, L. T. (2014). Fatores associados às recidivas de malária causada por Plasmodium vivax no Município de Porto Velho, Rondônia, Brasil , 2009. Cad. Saúde Pública, Rio de Janeiroa, 30(7), 1-15. https://doi.org/10.1590/S0074-02762007005000051

Venkatesan, M., Gadalla, N. B., Stepniewska, K., Dahal, P., Nsanzabana, C., Moriera, C., ... 
737

738

739

740

741

742

743

744

745

746

747

748

749

750

751

752

753

754

755

756

757

758

759

760

761

762

763

764

765

766

767

Asaq Molecular Marker Study Group, for the W. A. and A. M. M. S. (2014).

Polymorphisms in Plasmodium falciparum chloroquine resistance transporter and multidrug resistance 1 genes: parasite risk factors that affect treatment outcomes for P. falciparum malaria after artemether-lumefantrine and artesunate-amodiaquine. The American Journal of Tropical Medicine and Hygiene, 91(4), 833-843. https://doi.org/10.4269/ajtmh.14-0031

Wang, X., Mu, J., Li, G., Chen, P., Guo, X., Fu, L., ... Wellems, T. E. (2005). Decreased prevalence of the Plasmodium falciparum chloroquine resistance transporter 76T marker associated with cessation of chloroquine use against P. falciparum malaria in Hainan, People's Republic of China. American Journal of Tropical Medicine and Hygiene, 72(4), 410-414. https://doi.org/72/4/410 [pii]

Wasunna, B., Okiro, E. A., Webster, J., Todd, J., Snow, R. W., \& Jones, C. (2015). The impact of a community awareness strategy on caregiver treatment seeking behaviour and use of artemether-lumefantrine for febrile children in rural Kenya. PLoS ONE, 10(7), 1-19. https://doi.org/10.1371/journal.pone.0130305

Watsierah, C. A., Onyango, R. O., Ombaka, J. H., Abong 'o, B. O., \& Ouma, C. (2012). Provider knowledge of treatment policy and dosing regimen with artemether-lumefantrine and quinine in malaria-endemic areas of western Kenya. Malaria Journal, 11. https://doi.org/10.1186/1475-2875-11-436

Wellems, T. E., \& Plowe, C. V. (2001). Chloroquine-resistant malaria. Journal of Infectious Diseases, 184(6), 770-776. https://doi.org/10.1086/322858

Wesolowski, A., Eagle, N., Tatem, A. J., Smith, D. L., Noor, A. M., Snow, R. W., \& Buckee, C. O. (2012). Quantifying the Impact of Human Mobility on Malaria. Science, 338(6104), 267-270. https://doi.org/10.1126/science.1223467

White, N. J. (2011). Determinants of relapse periodicity in Plasmodium vivax malaria Determinants of relapse periodicity in Plasmodium vivax malaria. Malaria Journal, 297(October).

Whittaker, M. a, Dean, A. J., \& Chancellor, A. (2014). Advocating for malaria elimination learning from the successes of other infectious disease elimination programmes. Malaria Journal, 13(1), 221. https://doi.org/10.1186/1475-2875-13-221

WHO. (2007). Gender, Health and malaria. Retrieved from http://www.who.int/gender-equityrights/knowledge/gender-health-malaria.pdf 
768 WHO. (2009). Methods for surveillance of antimalarial drug efficacy. In WHO.

769 https://doi.org/ISBN 9789241597531

770 WHO. (2015). Malaria in Pregnant Women. Retrieved January 19, 2017, from

771 https://www.who.int/malaria/areas/high_risk_groups/pregnancy/en/

772 WHO. (2016a). Malaria. Retrieved November 16, 2016, from

$773 \mathrm{http}: / / \mathrm{www} . w h o . i n t / m e d i a c e n t r e /$ factsheets/fs094/en/

774 WHO. (2016b). Malaria. Retrieved November 9, 2016, from

775 http://www.who.int/mediacentre/factsheets/fs094/en/

776 WHO. (2018). World Malaria Report. 2018. ISBN 978924156469 4. https://doi.org/ISBN 978

7779241564403

778 Wiese, M. (2012). Integrated approaches to malaria control - addressing new challenges to

779 malaria research. Malaria Journal. https://doi.org/10.1186/1475-2875-11-s1-p104

780 Witkowski, B., Khim, N., Chim, P., Kim, S., Ke, S., Kloeung, N., ... Menard, D. (2013).

781 Reduced artemisinin susceptibility of plasmodium falciparum ring stages in western

782 cambodia. Antimicrobial Agents and Chemotherapy, 57(2), 914-923.

783 https://doi.org/10.1128/AAC.01868-12

784 Woodrow, C. J., \& White, N. J. (2017, January 1). The clinical impact of artemisinin resistance

785 in Southeast Asia and the potential for future spread. FEMS Microbiology Reviews, Vol. 41,

786 pp. 34-48. https://doi.org/10.1093/femsre/fuw037

787 Zhao, Y., Liu, Z., Soe, M. T., Wang, L., Soe, T. N., Wei, H., .. Cui, L. (2019). Genetic

788 Variations Associated with Drug Resistance Markers in Asymptomatic Plasmodium

789 falciparum Infections in Myanmar. Genes, 10(9), 692.

$790 \quad$ https://doi.org/10.3390/genes10090692

791 Zhong, D., Afrane, Y., Githeko, A., Cui, L., Menge, D. M., \& Yan, G. (2008). Molecular

792 epidemiology of drug-resistant malaria in western Kenya highlandshighlands. BioMed Ce,

793 7. https://doi.org/10.1186/1471-2334-8-105

794 


\section{Figure 1}

Map of Kenya showing the different malaria endemicity zones and locations of various surveillance hospitals.

Lake endemic region: Kisumu East and Kisumu West District Hospitals in Kisumu county, Semi-Arid, seasonal: Marigat District hospital in Baringo county and Isiolo District Hospital in Isiolo county. Coast endemic: Malindi District Hospital, Kilifi County. High land Epidemic: Kisii teaching and referral Hospital and Kericho District Hospital (QGIS version 2.18).

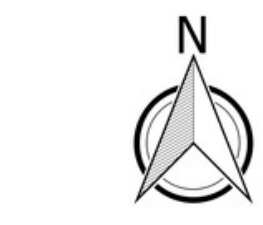

Key

Ocean

Lakes

Malaria Epidemic Zones

Coast Endemic

Highland Epidemic

Lake Endemic

Low Risk

Semi-Arid Seasonal

$\begin{array}{llllll}100 & 0 & 100 & 200 & 300 & 400 \\ \end{array}$

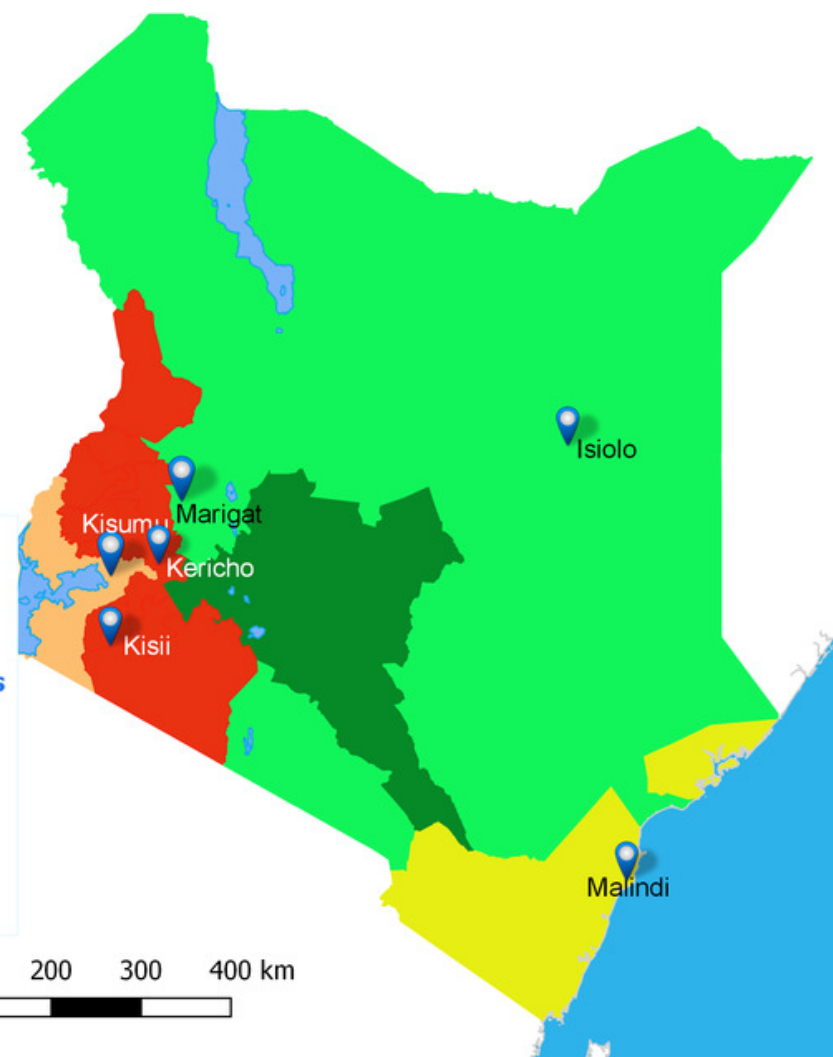

African Countries

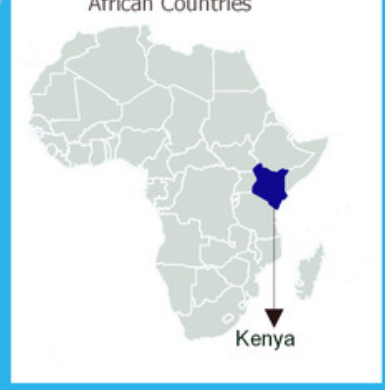


Figure 2

Comparison of parasite density by malaria epidemic zones.

Parasite density (natural log transformation). Dots represent outliers. P value $<0.05$ was considered significant.

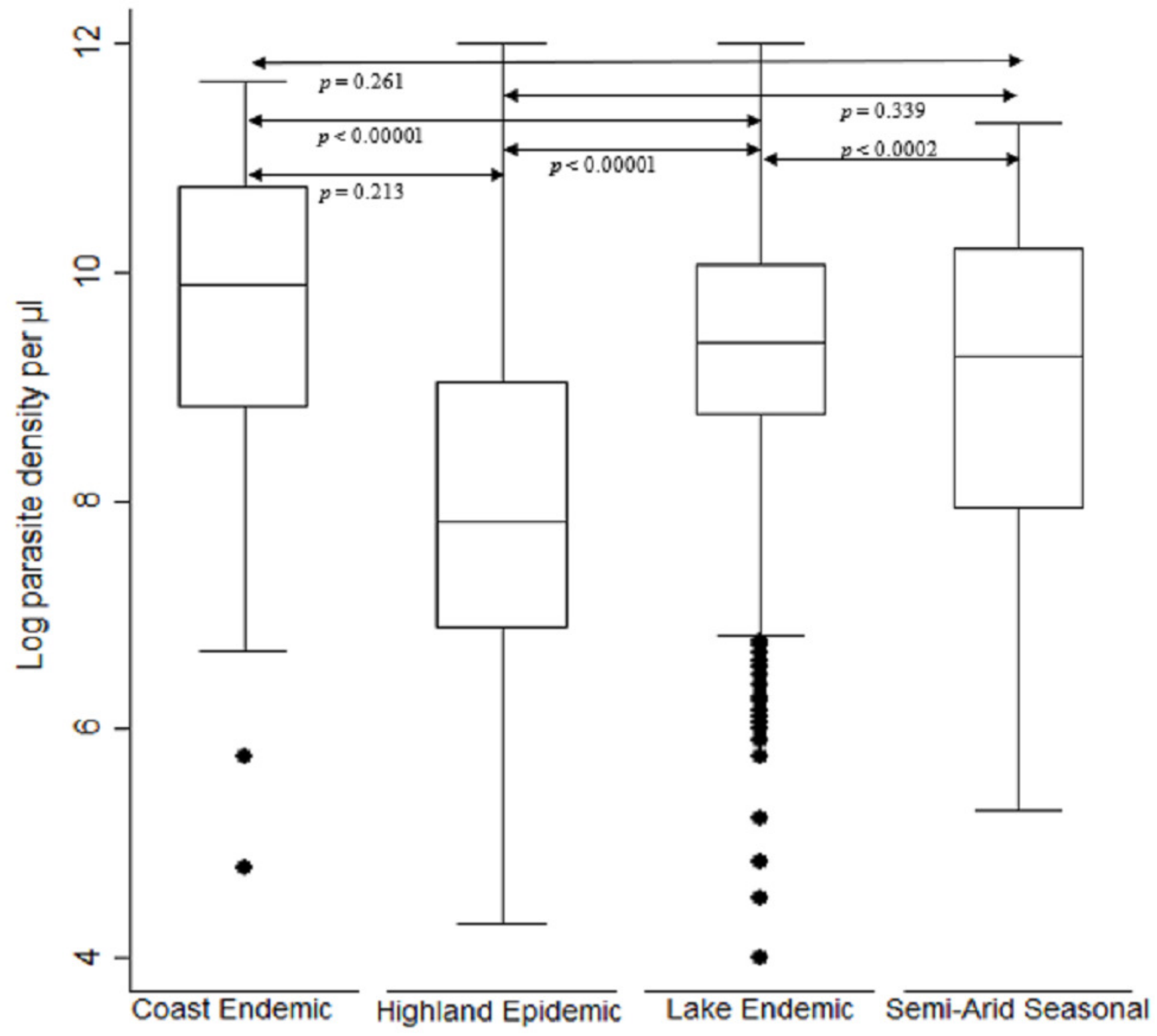


Figure 3

Comparison of parasite densities by age groups.

Parasite density (natural log transformation). Dots represent outliers. P value $<0.05$ was considered significant.

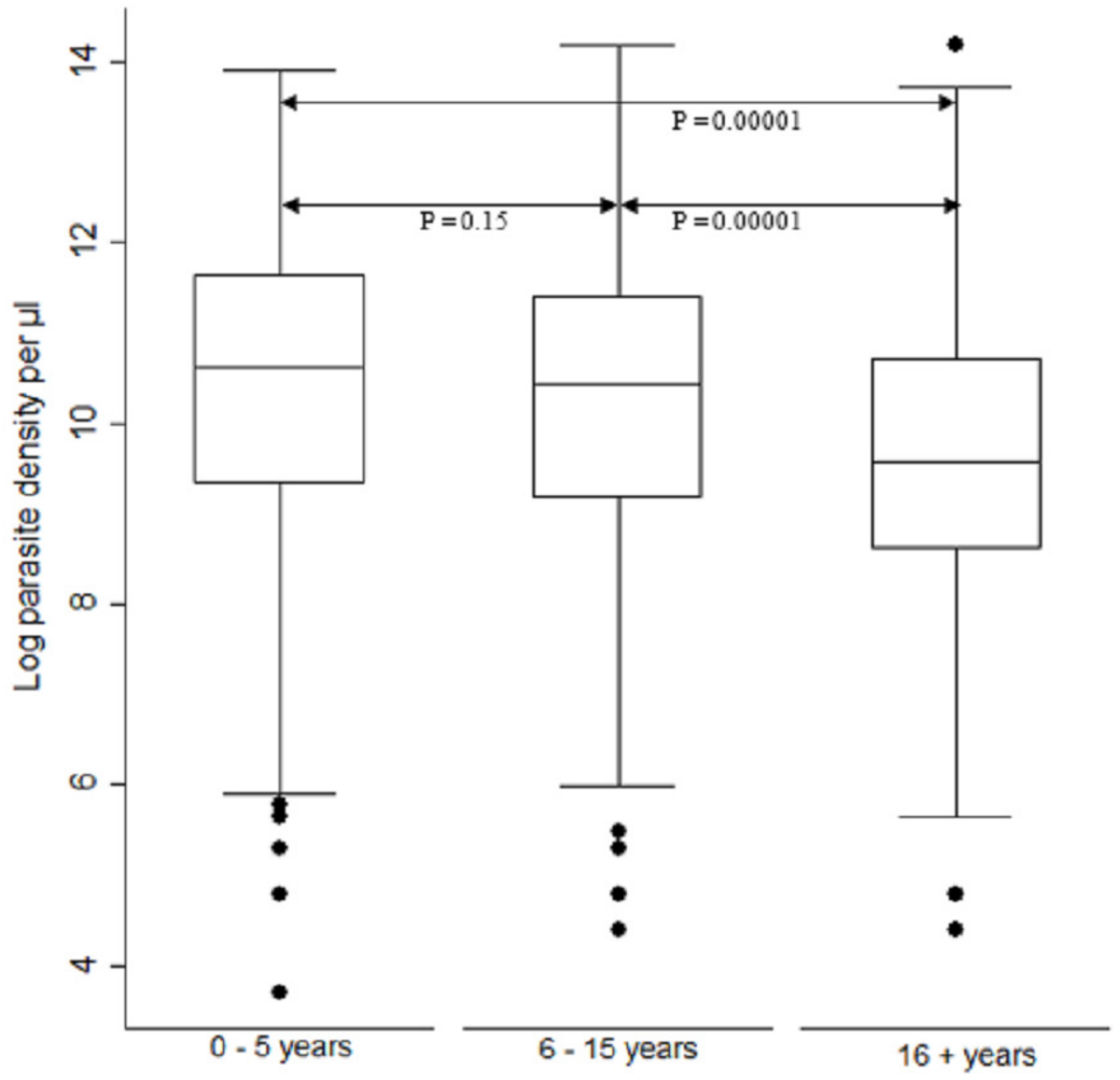




\section{Figure 4}

Trends of genotype frequencies of Pfcrt 76T, Pfmdr1 184F and Pfmdr1 86Y polymorphisms between 2008 and 2014.

There was a significant difference $(p<0.0001)$ for each of the Pfcrt 76T, Pfmdr1 $184 \mathrm{~F}$ and Pfmdr1 86Y SNPs with $\chi^{2}$ of $354,780,103$ respectively from 2008 to 2014. Pfcrt $76(n=1579)$, Pfmdr1 $86(\mathrm{n}=1676)$ and Pfmdr1 $184(1570)$.

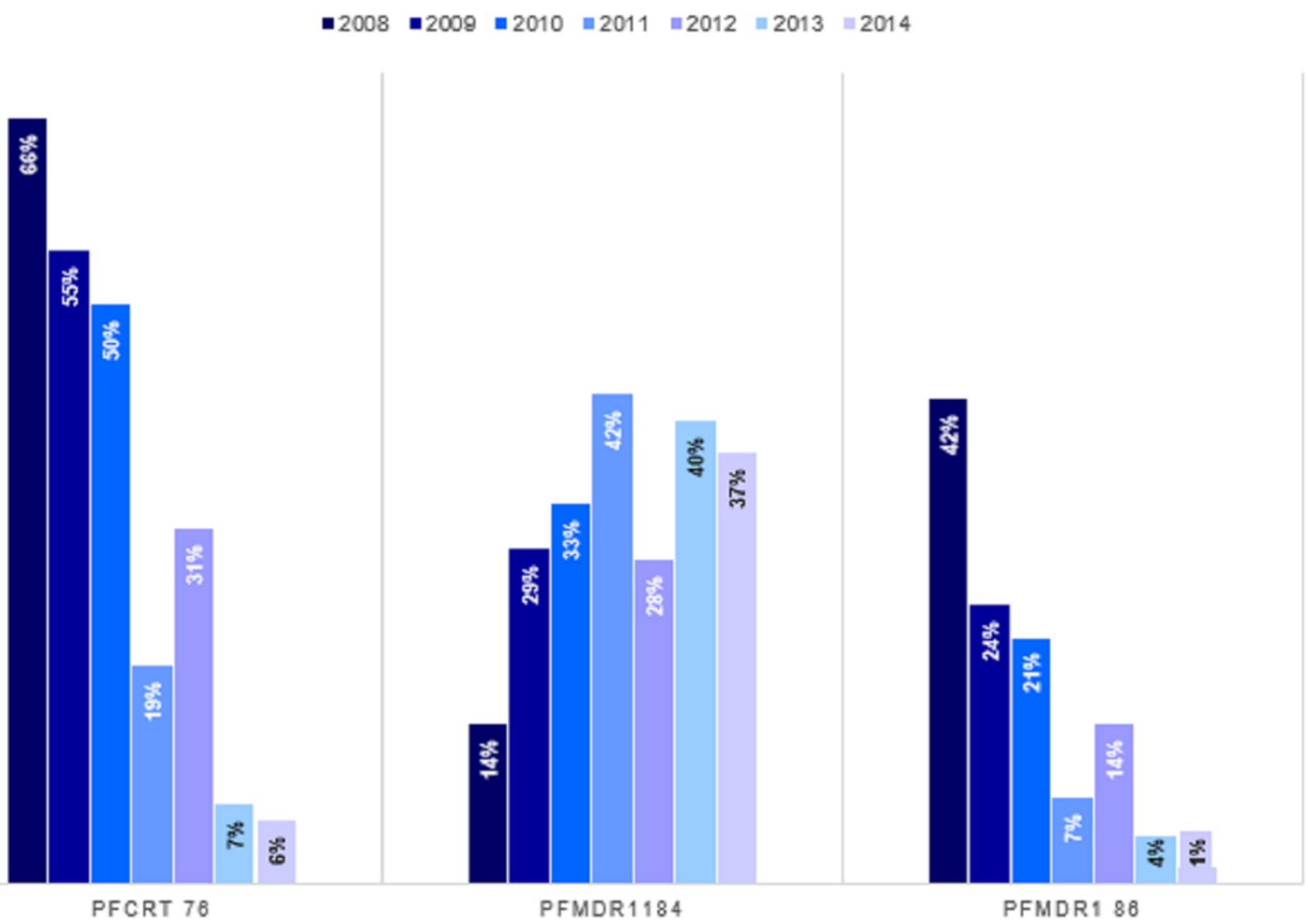




\section{Figure 5}

Spatial distribution patterns of A) Pfcrt 76T, B) Pfmdr1 184F and C) Pfmdr1 86Y SNPs by malaria epidemic zones using ArcGIS version 10.

There was a significant difference among the Pfcrt 76T, Pfmdr1 184F and Pfmdr1 86Y SNPs across the epidemic zones $(\chi 2(6)=39.5, p<0.0001),(\chi 2(6)=21.9, p<0.001)$ and $(\chi 2(6)$ $=17.1, p<0.009)$ respectively.

A

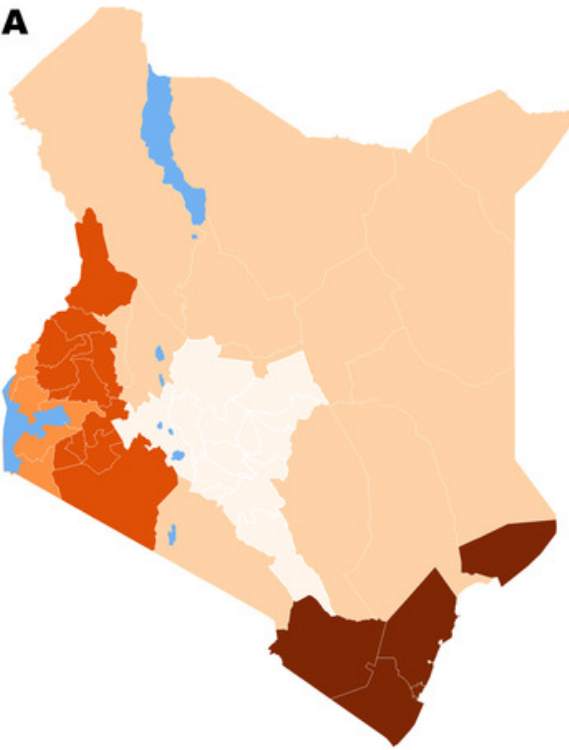

Key

Lakes

Pfcrt 76T SNP

No data : Low Risk

$5.0 \%$ : Semi-Arid Seasonal

9.3\% : Lake Endemic

$11.2 \%$ : Highland Epidemic

$34.8 \%$ : Coast Endemic

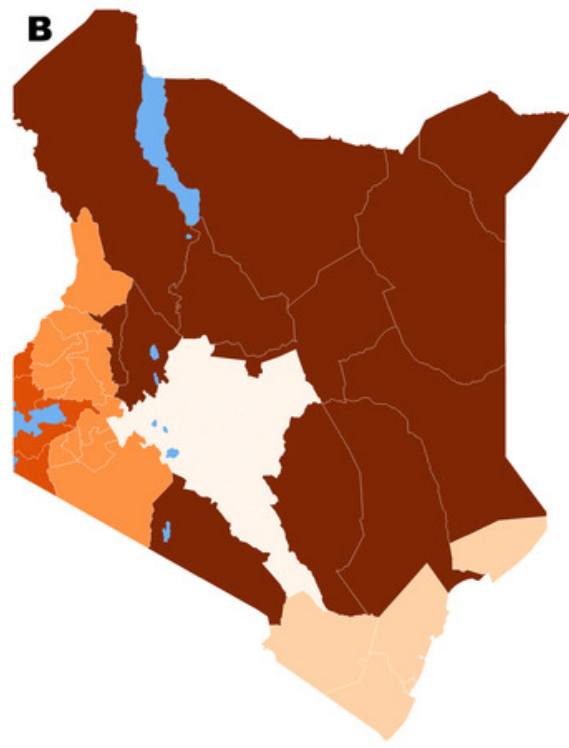

Key

Lakes

Pfmdr1 184F SNP

No data : Low Risk

$13.0 \%$ : Coast Endemic

36.8\% : Highland Epidemic

$38.4 \%$ : Lake Endemic

$45.0 \%$ : Semi-Arid Seasonal

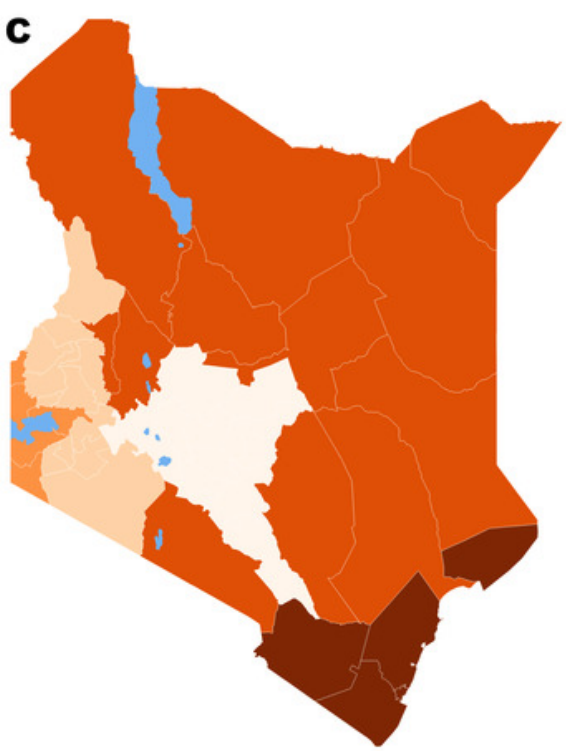

Key

Lakes

Pfmdr1 86Y SNP

No data : Low Risk

2.2\% : Highland Epidemic

3.3\% : Lake Endemic

$10.0 \%$ : Semi-Arid Seasonal

$30.4 \%$ : Coast Endemic 


\section{Table $\mathbf{1}$ (on next page)}

Demographic and clinical characteristics of subjects enrolled by resistance.

** Not applicable for chi-square test. $P$ value $<0.05$ was considered significant. SD- standard deviation. 
1

\begin{tabular}{|c|c|c|c|c|c|}
\hline \multirow[b]{2}{*}{ Characteristics } & \multicolumn{2}{|c|}{ Malaria Resistant Status } & \multirow[b]{2}{*}{$\begin{array}{c}\text { Total n (\%) } \\
\text { n=1,776 }\end{array}$} & \multirow[b]{2}{*}{ Chi Square } & \multirow[b]{2}{*}{ p - value } \\
\hline & $\begin{array}{l}\text { Resistant } n \\
(\%) \quad n=941\end{array}$ & $\begin{array}{l}\text { Non-Resistant n } \\
\quad(\%) n=835\end{array}$ & & & \\
\hline \multicolumn{6}{|l|}{ Sex } \\
\hline Male & $472(54.4 \%)$ & $395(45.6 \%)$ & $867(48.8 \%)$ & 1.44 & 0.23 \\
\hline Female & $469(51.6 \%)$ & $440(48.4 \%)$ & $909(51.2 \%)$ & & \\
\hline \multicolumn{6}{|l|}{ Age group, years } \\
\hline$\leq 5$ & $520(52.1 \%)$ & $479(47.9 \%)$ & $999(56.7 \%)$ & 0.4 & 0.82 \\
\hline $6-15$ & $206(53.4 \%)$ & $180(46.6 \%)$ & $386(21.9 \%)$ & & \\
\hline$\geq 16$ & $202(53.7 \%)$ & $174(46.3 \%)$ & $376(21.3 \%)$ & & \\
\hline $\begin{array}{l}\text { Mean body } \\
\text { temperature } \pm \text { SD }\end{array}$ & $38.1 \pm 1.24$ & $38.1 \pm 1.18$ & $38.1 \pm 1.21$ & $* *$ & $* *$ \\
\hline \multicolumn{6}{|c|}{ Ever travelled in the last 2 months } \\
\hline Yes & $368(55.3 \%)$ & $297(44.7 \%)$ & $665(37.5 \%)$ & 2.36 & 0.125 \\
\hline $\begin{array}{l}\quad \text { No } \\
\text { Ever had } \\
\text { malaria }\end{array}$ & $572(51.6 \%)$ & $537(48.4 \%)$ & $1,109(62.5 \%)$ & & \\
\hline Yes & $746(52.5 \%)$ & $676(47.5 \%)$ & $1,422(80.2 \%)$ & 0.79 & 0.372 \\
\hline No & $194(55.1 \%)$ & $158(44.9 \%)$ & $352(19.8 \%)$ & & \\
\hline \multicolumn{6}{|c|}{ Treated for malaria in the last 6 weeks } \\
\hline Yes & $118(62.8 \%)$ & $70(37.8 \%)$ & $188(10.6 \%)$ & 8.07 & 0.004 \\
\hline No & $822(51.8 \%)$ & $764(48.2 \%)$ & $1,586(89.4 \%)$ & & \\
\hline \multicolumn{6}{|l|}{ Chief Complain } \\
\hline Fever & $611(52.5 \%)$ & $552(47.5 \%)$ & $1,163(66.2 \%)$ & 0.46 & 0.978 \\
\hline Headache & $200(54.1 \%)$ & $170(45.9 \%)$ & $370(21.1 \%)$ & & \\
\hline Coughing & $10(55.6 \%)$ & $8(44.4 \%)$ & $18(1.0 \%)$ & & \\
\hline Joint pains & $20(50.0 \%)$ & $20(50.0 \%)$ & $40(2.3 \%)$ & & \\
\hline Others & $87(52.4 \%)$ & $79(47.6 \%)$ & $166(9.4 \%)$ & & \\
\hline
\end{tabular}

2 


\section{Table 2 (on next page)}

Distribution of Pfcrt 76, Pfmdr1 86 and 184 single nucleotide polymorphisms by zone.

Notes: Samples with mutant, wild-type and a mixture of mutant/wild-type were identified for each epidemic zone. Undetermined samples contained neither mutant, Wild-type nor a mixture of mutant/Wild-type. $P<0.05$ was considered significant. 


\begin{tabular}{|c|c|c|c|c|c|c|c|}
\hline $\begin{array}{l}\text { Polymorphisms } \\
(\mathrm{n}=1,676)\end{array}$ & $\begin{array}{l}\text { Lake } \\
\text { Endemic } \\
\end{array}$ & $\begin{array}{c}\text { Highland } \\
\text { Epidemic }\end{array}$ & $\begin{array}{l}\text { Semi-Arid } \\
\text { Seasonal }\end{array}$ & $\begin{array}{l}\text { Coast } \\
\text { Endemic } \\
\end{array}$ & Total & $\begin{array}{l}\text { Chi } \\
\text { Square }\end{array}$ & p - value \\
\hline \multicolumn{8}{|l|}{ Pfcrt-76 } \\
\hline Mutant & $302(29.6 \%)$ & $129(26.2 \%)$ & $3(11.1 \%)$ & $12(31.6 \%)$ & $446(28.3 \%)$ & 39.5 & 0.0001 \\
\hline Wild-type & $467(45.7 \%)$ & $282(57.2 \%)$ & $21(77.8 \%)$ & $10(26.3 \%)$ & $780(49.4 \%)$ & & \\
\hline $\begin{array}{l}\text { Wild- } \\
\text { type/mutant }\end{array}$ & $252(24.7 \%)$ & $82(16.6 \%)$ & $3(1.1 \%)$ & $16(42.1 \%)$ & $353(22.3 \%)$ & & \\
\hline \multicolumn{8}{|l|}{ Pfmdr1-86 } \\
\hline Mutant & $155(14.4 \%)$ & $56(10.8 \%)$ & $3(12.5 \%)$ & $15(27.3 \%)$ & $229(13.7 \%)$ & 21.9 & 0.001 \\
\hline Wild-type & $769(71.5 \%)$ & $415(79.7 \%)$ & $19(79.2 \%)$ & $35(63.6 \%)$ & $1,238(73.9 \%)$ & & \\
\hline $\begin{array}{l}\text { Wild- } \\
\text { type/mutant }\end{array}$ & $152(14.1 \%)$ & $50(9.6 \%)$ & $2(8.3 \%)$ & $5(9.1 \%)$ & $209(12.5 \%)$ & & \\
\hline \multicolumn{8}{|l|}{ Pfmdr1 184} \\
\hline Mutant & $319(33.1 \%)$ & $168(32.3 \%)$ & $13(32.5 \%)$ & $7(13.5 \%)$ & $507(32.3 \%)$ & 17.1 & 0.009 \\
\hline $\begin{array}{l}\text { Wild-type } \\
\text { Wild- }\end{array}$ & $434(45.0 \%)$ & $255(49.0 \%)$ & $13(32.5 \%)$ & $37(71.2 \%)$ & $739(47.1 \%)$ & & \\
\hline type/mutant & $212(21.9 \%)$ & $97(18.7 \%)$ & $7(17.5 \%)$ & $8(15.4 \%)$ & $324(20.6 \%)$ & & \\
\hline
\end{tabular}

2 


\section{Table 3(on next page)}

Association of demographic and clinical factors with CQR.

Ref- Reference group, OR- Odds ratio, $\mathrm{Cl}$-confidence interval. $P<0.05$ was considered significant. 
1

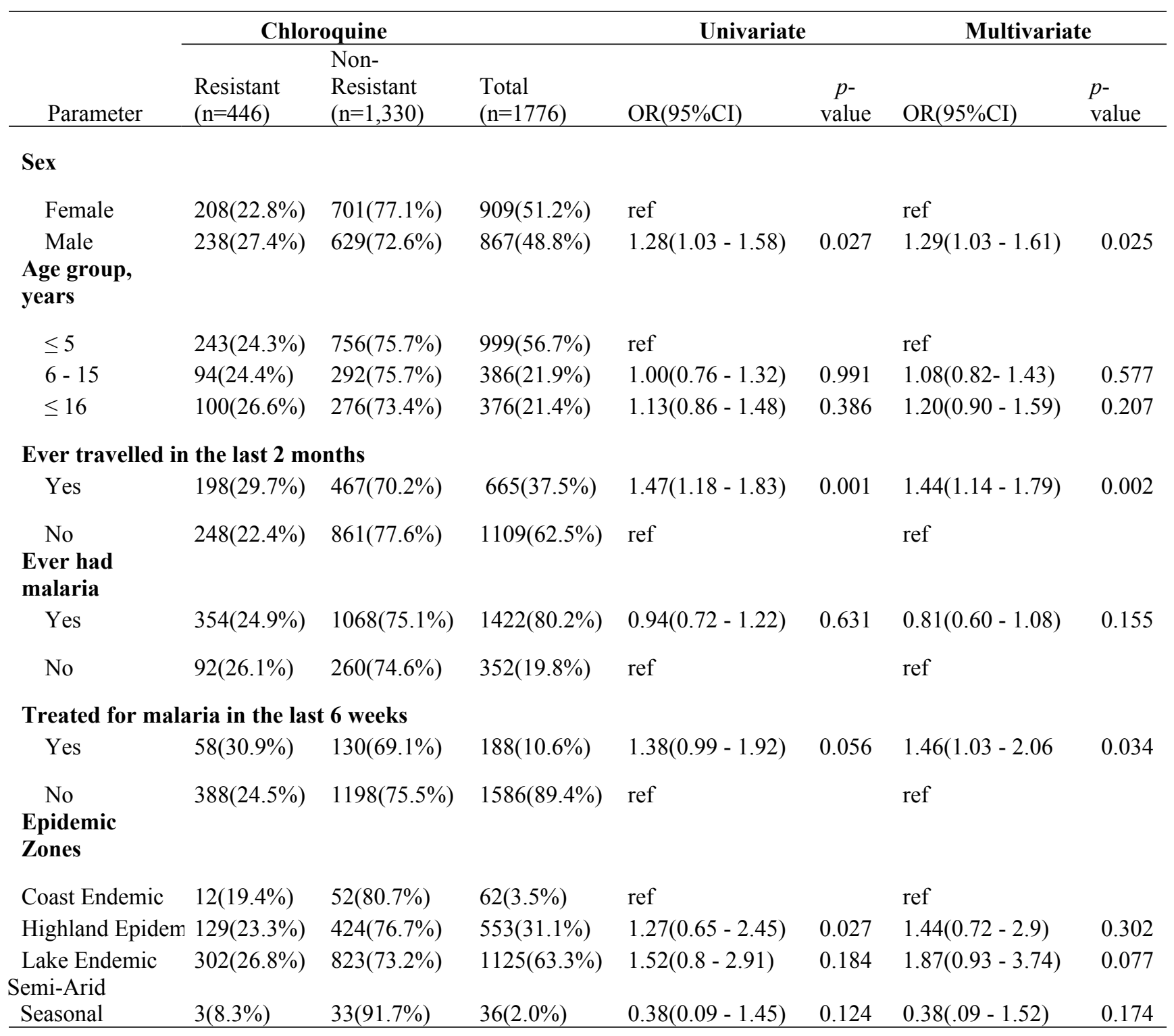

2 